\title{
A study of the population of LMXBs in the bulge of M $31^{\star}$
}

\author{
R. Voss ${ }^{1}$ and M. Gilfanov ${ }^{1,2}$ \\ ${ }^{1}$ Max Planck Institut für Astrophysik, Karl-Schwarzschild-Str. 1, 85740 Garching bei München, Germany \\ e-mail: [voss;gilfanov] ampa-garching .mpg.de \\ 2 Space Research Institute, Russian Academy of Sciences, Profsoyuznaya 84/32, 117997 Moscow, Russia
}

Received 21 October 2006 / Accepted 27 February 2007

ABSTRACT

\begin{abstract}
Aims. We explore the population of X-ray point sources in the bulge of M 31 to contrast properties of various subpopulations, such as persistent and transient sources and primordial LMXBs and dynamically formed ones.

Methods. Based on the data from 26 archival Chandra observations we study the source content and properties of various subpopulations of X-ray sources to a maximum distance of $12^{\prime}$ from the centre of M 31 .

Results. To a limiting luminosity of $\sim 10^{35} \mathrm{erg} \mathrm{s}^{-1}$ we find $263 \mathrm{X}$-ray point sources, with $\sim 1 / 3$ of these being background galaxies. A study of the spatial distribution and the luminosity function of the X-ray sources shows that the distribution of primordial LMXBs is consistent with the distribution of the $K$-band light and that their luminosity function flattens below $\sim 10^{37} \mathrm{erg} \mathrm{s}^{-1}$ to the $\mathrm{d} N / \mathrm{d} L \propto L^{-1}$ law in agreement with the behaviour found earlier for LMXBs in the Milky Way and in Cen A. Within a radius of $12^{\prime}$, the luminosity function is independent of distance to the centre of M31, in contrast to earlier Chandra studies. The LMXBs located in globular clusters and within $\sim 1^{\prime}$ from the centre of M31 are presumably created via dynamical interactions. The dynamical origin of the $r<1^{\prime}$ sources is strongly suggested by their radial distribution which follows the $\rho_{*}^{2}$ profile rather than the K-band light distribution. Their luminosity function shows a prominent fall-off below $\log \left(L_{X}\right) \lesssim 36.5$. Although the statistics is insufficient to claim a genuine low-luminosity cut-off in the luminosity function, the best fit powerlaw with a slope of $-0.6 \pm 0.2$ is significantly flatter than the $\mathrm{d} N / \mathrm{d} L \propto L^{-1}$ law. We also searched for transients and found 28 sources that varied by a factor larger than 20 . Their spatial distribution follows the distribution of the persistent LMXBs within the accuracy allowed by the limited number of transients.
\end{abstract}

Key words. galaxies: individual: M 31 - X-rays: binaries - X-rays: galaxies

\section{1. introduction}

With the advent of Chandra, X-ray point sources in nearby galaxies became a subject of intense study (see Fabbiano 2006, and references therein). In spiral and starburst galaxies the X-ray luminosity function (LF) has been shown to be a powerlaw with a differential slope of $\sim 1.6$ (Grimm et al. 2003), whereas the LF in elliptical galaxies seems to have a more complicated shape, being steep at the bright end, $\log \left(L_{\mathrm{X}}\right)>37.5$, with power law index in the $\sim 1.8-2.5$ range, and flat below $\sim \log \left(L_{X}\right)<37.0$ (Gilfanov 2004; Voss \& Gilfanov 2006). There is, however, currently no consensus on the existence and position of the breaks, and on the slope below a few times $10^{37} \mathrm{erg} \mathrm{s}^{-1}$ (Kim, D.-W. et al. 2006).

While M31 is not an elliptical galaxy, the population of $\mathrm{X}$-ray sources in the bulge mainly consists of low mass X-ray binaries (LMXBs), similar to the population of X-ray sources in elliptical galaxies. It is therefore fair to expect the X-ray LF to be similar to that of ellipticals (and perhaps even more interesting if differences show up). The LF can only be studied to a limiting luminosity of $10^{37} \mathrm{erg} \mathrm{s}^{-1}$ in the closest large ellipticals (Kim, D.-W. et al. 2006), except for Cen A (Voss \& Gilfanov 2006) that is both an unusual elliptical and suffers from contamination of X-rays from other sources than binaries. In contrast to this, the proximity of M 31 makes it possible to study the LF down to $10^{35} \mathrm{erg} \mathrm{s}^{-1}$.

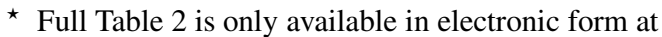
http://www. aanda.org
M 31 has been observed extensively both by XMM-Newton and Chandra, and the point source population has been analysed in a number of papers, see e.g. Kong et al. (2002); Kaaret (2002); Pietsch et al. (2005b); Williams et al. (2004a, 2006). In the central parts of M31 the point spread function (PSF) of XMM-Newton causes source confusion and therefore only Chandra observations are suited for studies of the weak sources in this region. The LF of the inner region of M 31 has previously been studied with Chandra by Kong et al. (2002, 2003); Williams et al. (2004a). There are several good reasons to reinvestigate the LF in this region. The inner bulge of M 31 has been observed a number of times after this study, significantly increasing the exposure, and also the previous study did not include the effects of incompleteness and contamination by background sources, which influences their conclusions significantly.

The paper is structured as follows. In Sect. 2 we describe the data sets and the basic data preparation and analysis. The identification of sources in other wavelengths is discussed in this section. The properties of the population of X-ray binaries in the bulge of M 31 are analysed in Sect. 3, including the spatial distribution and analysis of incompleteness effects. The search for and analysis of transient sources is presented in Sect. 4. The LFs of the source populations are analysed in Sect. 5. We conclude in Sect. 6.

\section{Data analysis}

The analysis in this paper is based on 26 Chandra ACIS observations, with aimpoints within $10^{\prime}$ from the centre of 
Table 1. The specifications of the Chandra observations used in this paper. The corrections given in the last two columns are the corrections applied to the aspect files to align the observations and to achieve absolute astrometry. 1 pixel equals $0.492^{\prime \prime}$.

\begin{tabular}{|c|c|c|c|c|c|c|c|c|}
\hline Obs-ID & Date & Instrument & Exp. Time & $\mathrm{RA}$ & Dec & Data Mode & Correction West & Correction North \\
\hline 0303 & 1999 Oct. 13 & ACIS-I & $12.01 \mathrm{ks}$ & 004244.4 & +411608.30 & FAINT & +0.72 pixel & -0.29 pixel \\
\hline 0305 & 1999 Dec. 11 & ACIS-I & $04.18 \mathrm{ks}$ & 004244.4 & +411608.30 & FAINT & -0.59 pixel & -0.16 pixel \\
\hline 0306 & 1999 Dec. 27 & ACIS-I & $04.18 \mathrm{ks}$ & 004244.4 & +411608.30 & FAINT & -0.51 pixel & -0.01 pixel \\
\hline 0307 & 2000 Jan. 29 & ACIS-I & $04.17 \mathrm{ks}$ & 004244.4 & +411608.30 & FAINT & -0.34 pixel & +0.19 pixel \\
\hline 0308 & 2000 Feb. 16 & ACIS-I & $04.06 \mathrm{ks}$ & 004244.4 & +411608.30 & FAINT & +0.77 pixel & +1.34 pixel \\
\hline 0309 & 2000 Jun. 01 & ACIS-S & $05.16 \mathrm{ks}$ & 004244.4 & +411608.30 & FAINT & -0.41 pixel & +0.12 pixel \\
\hline 0310 & 2000 Jul. 02 & ACIS-S & $05.14 \mathrm{ks}$ & 004244.4 & +411608.30 & FAINT & -0.40 pixel & +0.17 pixel \\
\hline 0312 & 2000 Aug. 27 & ACIS-I & $04.73 \mathrm{ks}$ & 004244.4 & +411608.30 & FAINT & -0.90 pixel & +1.62 pixel \\
\hline 1575 & 2001 Oct. 05 & ACIS-S & $38.15 \mathrm{ks}$ & 004244.4 & +411608.30 & FAINT & -0.97 pixel & +0.04 pixel \\
\hline 1577 & 2001 Aug. 31 & ACIS-I & $04.98 \mathrm{ks}$ & 004308.5 & +411820.00 & FAINT & -2.79 pixel & -2.71 pixel \\
\hline 1581 & 2000 Dec. 13 & ACIS-I & $04.46 \mathrm{ks}$ & 004244.4 & +411608.30 & FAINT & -0.62 pixel & +2.93 pixel \\
\hline 1582 & 2001 Feb. 18 & ACIS-I & $04.36 \mathrm{ks}$ & 004244.4 & +411608.30 & FAINT & +1.65 pixel & +2.49 pixel \\
\hline 1583 & 2001 Jun. 10 & ACIS-I & $05.00 \mathrm{ks}$ & 004244.4 & +411608.30 & FAINT & -0.50 pixel & -3.95 pixel \\
\hline 1585 & 2001 Nov. 19 & ACIS-I & $04.95 \mathrm{ks}$ & 004305.6 & +411703.30 & FAINT & -1.28 pixel & -0.81 pixel \\
\hline 2896 & 2002 Feb. 06 & ACIS-I & $04.97 \mathrm{ks}$ & 004305.5 & +411703.30 & FAINT & -0.20 pixel & +0.83 pixel \\
\hline 2897 & 2002 Jan. 08 & ACIS-I & $04.97 \mathrm{ks}$ & 004309.8 & +411900.72 & FAINT & -0.44 pixel & +0.01 pixel \\
\hline 2898 & 2002 Jun. 02 & ACIS-I & $04.96 \mathrm{ks}$ & 004309.8 & +411900.72 & FAINT & -0.56 pixel & -0.02 pixel \\
\hline 4360 & 2002 Aug. 11 & ACIS-I & $04.97 \mathrm{ks}$ & 004244.4 & +411608.90 & FAINT & -0.19 pixel & -0.07 pixel \\
\hline 4678 & 2003 Nov. 09 & ACIS-I & $04.87 \mathrm{ks}$ & 004244.4 & +411608.90 & FAINT & +0.06 pixel & -0.35 pixel \\
\hline 4679 & 2003 Nov. 26 & ACIS-I & $04.77 \mathrm{ks}$ & 004244.4 & +411608.90 & FAINT & +0.00 pixel & -0.96 pixel \\
\hline 4680 & 2003 Dec. 27 & ACIS-I & $05.24 \mathrm{ks}$ & 004244.4 & +411608.90 & FAINT & -0.36 pixel & -0.86 pixel \\
\hline 4681 & 2004 Jan. 31 & ACIS-I & $05.13 \mathrm{ks}$ & 004244.4 & +411608.90 & FAINT & -0.59 pixel & -1.12 pixel \\
\hline 4682 & 2004 May 23 & ACIS-I & $04.93 \mathrm{ks}$ & 004244.4 & +411608.90 & FAINT & -0.64 pixel & -0.07 pixel \\
\hline
\end{tabular}

M 31 (RA 0042 44.31, Dec +41 16 09.4). Information about the observations is listed in Table 1 . The data preparation was done following the standard $\mathrm{CIAO}^{1}$ threads (CIAO version 3.2.1; CALDB version 3.0.3), and limiting the energy range to $0.5-8.0 \mathrm{keV}$. The ACIS chips sometimes experience flares of enhanced background. For point source detection and luminosity estimation it is not necessary to filter out the flares, since the increased exposure time outweighs the increased background.

We used CIAO wavdetect to detect sources. The input parameters for the detection procedure are similar to those used in Voss \& Gilfanov (2006). We detected sources within $10^{\prime}$ of the aimpoint in each of the individual observations. Due to limitations of the absolute astrometry of Chandra, the observations have to be aligned before they are combined. We chose to align the observations to OBS-ID 1575, as this is the observation with highest exposure time. For each of the observations we determined the number of sources matching sources found in OBSID 1575, excluding all ambiguous matches, such as a source in one of the source lists being close to two sources in the other list. The source lists were shifted relative to each other, and the smallest rms-distance between the sources were found. This method made it possible to align the 25 observations with OBS-ID 1575 with a minimum of 40 matches. The corrections applied to the observations are listed in Table 1. All the observations were then reprojected into the coordinate systems of OBS-ID 1575 using CIAO reproject_events, and a merged observations were created. Notice that the steps above were taken in order to align and combine the observations, not to achieve good absolute astrometry. This will be dealt with using optical data in Sect. 2.2.

An exposure map was created for each of the observations, assuming the energy distribution to be a powerlaw with photon index of 1.7 and Galactic absorption of $6.68 \times 10^{20} \mathrm{~cm}^{-2}$ (Dickey \& Lockman 1990). In the following we use the same spectrum

\footnotetext{
${ }^{1}$ http://cxc.harvard.edu/ciao/
}

to convert the observed count rates to unabsorbed source flux. To calculate the luminosity of the sources, we assumed a distance of $780 \mathrm{kpc}$ to M 31 (Stanek \& Garnavich 1998; Macri 2001).

To estimate the source counts we applied circular aperture photometry. The output count rate for each detected source is calculated inside a circular region centered on the source central coordinates given by wavdetect. The radius of the circle was determined individually for each source so that the encircled energy was $85 \%$. To find this radius we extracted the PSF using CIAO psfextract task for each of the 26 observations, and the PSFs were combined using the values of the exposure maps as weights. For the background region we used a circle with radius 3 times the radius of the source region and the source region excluded, as well as the source regions of eventual nearby sources. The corrected source counts and errors were then found by the equations (Harnden et al. 1984):

$$
S=\frac{C(b-d) d^{-1}-Q}{\alpha b d^{-1}-\beta}
$$

and

$$
\sigma_{S}^{2}=\frac{\sigma_{C}^{2}(b-d)^{2} d^{-2}+\sigma_{Q}^{2}}{\left(\alpha b d^{-1}-\beta\right)^{2}} .
$$

Here $S$ is the total number of counts from the source, $C$ is the number of counts inside the source region and $Q$ is the number of counts in the background region, $\alpha$ is the integral of the PSF over the source region, $\beta$ is the integral of the PSF over the source and background regions, $b$ is the area of the source and background regions and $d$ is the area of the source region. For close sources the extraction regions can overlap. In this case a second iteration was performed. In this iteration the number of counts of the neighbouring sources, together with their PSF was used to find the contamination from nearby sources of the source 
and background regions of a source. This contamination from nearby sources was then subtracted from $C$ and $Q$, and Eq. (1) was repeated. In all cases the effect of the contamination was small enough to justify the use of this method with only on iteration.

\section{1. $2 M A S S ~ L G A$}

To compare the spatial distribution of the point sources with the distribution of mass in M31, we used a $K$-band image of the region from the 2MASS Large Galaxy Atlas (Jarret et al. 2003). The image has a resolution of $1^{\prime \prime}$, and it is therefore possible to clearly see point sources. While many of the sources are objects inherent to M31, such as globular clusters, some of the sources may be foreground or background objects. In order to remove these objects, we correlate the image with the Revised Bologna catalogue of M31 globular clusters (Galleti et al. 2004), and thereby find the maximum $K$-band luminosity of a globular cluster, $\sim 2 \times 10^{6} L_{K, \odot}$. All point sources more luminous than this are removed from the image (replaced with local background). This corresponds to 14 per cent of the luminosity in the outer part of the image (the annulus $9^{\prime}-12^{\prime}$ ). Point sources with luminosities between $\sim 4 \times 10^{5} L_{K, \odot}$ and $\sim 2 \times 10^{6} L_{K, \odot}$ corresponds only to 2 per cent of the total luminosity, and most of these sources are GCs in M 31. We therefore conclude that after our removal of the most luminous sources, contamination from point sources not in M31 is insignificant in the outer region. In the rest of the image the luminosity density of M31 is higher and contamination is therefore even less important.

\subsection{Absolute astrometry}

We used the 2MASS All-Sky Point Source Catalog (Skrutskie et al. 2006) to achieve better absolute astrometry. This catalog was chosen, due to the high number of (true) matches with our source list. The astrometric precision ranges from $\sim 0.1^{\prime \prime}$ for brighter sources to $0.4^{\prime \prime}$ for the weakest sources. The X-ray sources were correlated with the 3132 2MASS point sources in the observed region and the X-ray image was shifted to give the smallest rms-distance for matches with a distance less than $1^{\prime \prime}$. This gives a correction of -0.97 pixel west and +0.04 pixel north with 40 matches ( $<2$ random matches expected). We note that after the corrections, our source coordinates are in good agreement with the coordinates given by previous studies (Kong et al. 2002; Williams et al. 2004a).

\subsection{Source identifications}

For the identification of the detected sources we have used a variety of catalogues. For the identification of globular clustes we have used the Revised Bologna Catalogue of M 31 globular clusters (Galleti et al. 2004) as well as the lists given by Magnier (1993) and Fan et al. (2005). GCs were divided into the categories confirmed GCs and candidate GCs, following (Galleti et al. 2004), and the GCs in Magnier (1993) and Fan et al. (2005) were all considered candidates. Planetary nebulae have been identified using Ciardullo et al. (1989), and supernova remnants using Magnier et al. (1995). Furthermore we have searched for sources coincident with stellar novae in Pietsch et al. (2005a). Stars were identified using Galleti et al. (2004) as well as GSC 2.2 (Morrison et al. 2003) and USNO-B1 (Monet et al. 2003) (the latter two catalogues gave only sources found in Galleti et al. (2004) as well). Finally we searched for possible counterparts using the NASA/IPAC Extragalactic Database (NED) and SIMBAD. A source was assumed to be a counterpart to the X-ray source if within a distance of $2.5^{\prime \prime}$, except for supernova remnants for which the distance was $5^{\prime \prime}$.

A number of objects have been excluded from the following analysis, 4 foreground stars, 3 SNRs and one extended source (which was detected as two sources). More sources have been identified with objects, as can be seen from our source list, but the number is small, and the meaning of the identifications is uncertain, and we have therefore chosen to keep them in the sample. For example we note that a detailed study of the planetary nebulae correlations (Williams et al. 2004b) suggested that most are not true counterparts. Also of the four novae correlations, only one (source 128) is a true counterpart (W. Pietsch, private communication).

Our final source list consists of 263 sources within a radius of $12^{\prime}$ from the centre of M 31 (Table 2). Of these 9 sources are not included in the analysis below. Above $10^{37} \mathrm{erg} \mathrm{s}^{-1}$ there are 48 sources, and above $10^{36} \mathrm{erg} \mathrm{s}^{-1}$ (approximately the completeness limit), there are 136 sources included in the analysis. We expect $\sim 29$ of the sources with luminosity $>10^{36} \mathrm{erg} \mathrm{s}^{-1}$ and $\sim 89$ of all the sources to be background sources, taking into account incompleteness, see Sects. 3.1.3 and 3.3. We find that 15 sources are coincident with GCs ( 0.25 random matches expected) and 14 with GC candidates (1 random match expected).

\section{Populations of sources in the bulge of M 31}

\subsection{Expected numbers}

\subsubsection{Low mass $X$-ray binaries}

LMXBs are related to the population of old stars, and there is therefore a correlation between their number and the stellar mass of a galaxy (Gilfanov 2004). In order to estimate the expected number and luminosity distribution of LMXBs we used a $K$-band image from 2MASS Large Galaxy Atlas (Jarret et al. 2003) and integrated the flux emitted in the parts of M31 analysed in this paper (excluding luminous point sources not related to the galaxy, see Sect. 2.1). This gives a $K$-band luminosity of $L_{K}=4.4 \times 10^{10} L_{\odot}$. To convert it to the stellar mass we use the color-dependent $K$-band mass-to-light ratio from Bell \& De Jong (2001). For the extinction corrected optical color of the bulge of M31, $(B-V) \approx 0.95$ (Walterbos \& Kennicutt 1987), the massto-light ratio is $M_{*} / L_{K} \approx 0.85$. This gives the stellar mass of $3.8 \times 10^{10} M_{\odot}$, assuming that the absolute $K$-band magnitude of the sun is equal to $M_{K, \odot}=3.39$. Using the results of Gilfanov (2004) we predict $\approx 55.7$ LMXBs with $L_{X}>10^{37} \mathrm{erg} \mathrm{s}^{-1}$, and $\approx 128.9$ with $L_{\mathrm{X}}>10^{36} \mathrm{erg} \mathrm{s}^{-1}$.

\subsubsection{High mass $X$-ray binaries}

Being young objects, HMXBs are associated with star formation and, as expected for the bulge of a spiral galaxy, are by far a less significant contribution to the population of X-ray binaries than LMXBs. Star formation is mostly associated with the disk of M31. An investigation of the star formation rate of the disk has been conducted by Williams (2003), who find that the mean SFR over the last $60 \mathrm{Myr}$ for $1.4 \mathrm{deg}^{2}$ of the M 31 disk is $0.63 \pm 0.07 M_{\odot} \mathrm{yr}^{-1}$ (in the range $0.1-100 M_{\odot}$ ) with no drastic changes. Assuming a flat SFR density over the galaxy gives an $S F R$ of $\approx 0.048 M_{\odot} \mathrm{yr}^{-1}$ within the region analyzed in this paper. We used the calibration of Grimm et al. (2003) to calculate the expected number of HMXBs 
Table 2. The list of point like X-ray sources within a distance of $12^{\prime}$ from the centre of M31. The full table is available in the online version of the paper.

\begin{tabular}{|c|c|c|c|c|c|c|c|c|c|c|}
\hline $\begin{array}{l}\text { Number } \\
\text { (1) }\end{array}$ & $\begin{array}{c}\text { distance } \\
\text { (2) }\end{array}$ & $\begin{array}{l}\text { RA } \\
(3)\end{array}$ & $\begin{array}{l}\text { Dec } \\
\text { (4) }\end{array}$ & $\begin{array}{l}\text { cts } \\
(5)\end{array}$ & $\begin{array}{r}\text { corrected cts } \\
\text { (6) }\end{array}$ & $\begin{array}{r}\text { error } \\
(7)\end{array}$ & $\begin{array}{c}\text { luminosity } \\
\text { (8) }\end{array}$ & $\begin{array}{l}\text { type } \\
\text { (9) }\end{array}$ & $\begin{array}{l}\text { id } \\
\text { (10) }\end{array}$ & $\begin{array}{c}\text { name } \\
(11)\end{array}$ \\
\hline 1 & 1.0 & 004244.37 & 411608.7 & 2245 & 2580.7 & 58.0 & $7.08 \mathrm{e}+36$ & & & $\mathrm{r} 1-10$ \\
\hline 2 & 2.2 & 004244.38 & 411607.4 & 2864 & 3308.2 & 65.4 & $1.08 \mathrm{e}+37$ & & & r1-9 \\
\hline 3 & 4.0 & 004244.38 & 411605.4 & 1117 & 1233.9 & 41.3 & $5.32 \mathrm{e}+36$ & & & r1-21 \\
\hline 4 & 4.6 & 004244.30 & 411614.0 & 316 & 344.9 & 22.6 & $1.52 \mathrm{e}+36$ & & & r1-22 \\
\hline 5 & 5.4 & 004243.86 & 411611.1 & 225 & 244.1 & 19.2 & $1.07 \mathrm{e}+36$ & & & r1-27 \\
\hline 6 & 7.4 & 004243.87 & 411603.9 & 709 & 811.6 & 33.1 & $3.53 \mathrm{e}+36$ & & & r1-23 \\
\hline 7 & 9.7 & 004244.68 & 411618.2 & 906 & 1047.3 & 37.3 & $4.62 \mathrm{e}+36$ & & & $\mathrm{r} 1-8$ \\
\hline 8 & 10.6 & 004245.24 & 411611.1 & 456 & 516.2 & 26.8 & $2.25 e+36$ & & & r1-20 \\
\hline 9 & 14.5 & 004245.60 & 411608.6 & 1026 & 1191.0 & 39.5 & $5.21 e+36$ & & & r1-7 \\
\hline 10 & 15.3 & 004245.12 & 411621.7 & 3098 & 3631.2 & 67.9 & $1.61 e+37$ & RAD & SIM WSTB 37W135 & r1-4 \\
\hline 11 & 20.8 & 004243.88 & 411629.6 & 1293 & 1463.5 & 44.5 & $6.54 e+36$ & & & r1-11 \\
\hline 12 & 21.7 & 004246.01 & 411619.6 & 468 & 531.3 & 27.2 & $2.33 e+36$ & $\mathrm{t}$ & & r1-19 \\
\hline 13 & 23.8 & 004243.75 & 411632.4 & 2329 & 2726.7 & 59.0 & $1.24 \mathrm{e}+37$ & & & r1-12 \\
\hline 14 & 24.0 & 004242.18 & 411608.3 & 4525 & 5353.9 & 81.6 & $2.45 e+37$ & $\mathrm{t}$ & & $\mathrm{r} 1-5$ \\
\hline 15 & 25.9 & 004242.48 & 411553.7 & 3077 & 3601.1 & 67.6 & $1.62 \mathrm{e}+37$ & PN & CIA 4 & r1-14 \\
\hline
\end{tabular}

(1) - The sequence number; (2) - Distance to the centre in arcsec; (3),(4) - Right ascension and declination of source; (5) - Source counts; (6) - Source counts after background subtraction; (7) - Statistical error on source counts after background subtraction; (8) - X-ray luminosity, 0.5-8 keV, assuming $780 \mathrm{kpc}$ distance; (9) - Source Type: GC - confirmed globular cluster, GCC - globular cluster candidate, PN - planetary nebula, FGS - foreground star, NOVA - nova, EmO - emission line object, RAD - radio source, SNR - supernova remnant, EXT - extended source, $\mathrm{t}$ - transient source; (10) - precise identification and reference: Bol - Galleti et al. (2004), Fan - Fan et al. (2005), Mita - Magnier (1993), MLA - Meyssonnier et al. (1993), W2 - Williams et al. (2004b), CIA - Ciardullo et al. (1989), CFN - Ciardullo et al. (1987), PIE - Pietsch et al. (2005a), SI - Shafter \& Irby (2001), B68 - Börngen (1968), SIM - Simbad, GLG - Gelfand et al. (2004), B90 - Braun (1990), MG - Magnier et al. (1995), Cra - Crampton et al. (1984); (11) - Source name in Kong et al. (2002), Williams et al. (2004a) and Williams et al. (2006); Sources not included in these catalogues are marked with $\mathrm{K}$ if observed in Kaaret (2002), else with X, indicating that these are new sources.

(see comment in Shtykovskiy \& Gilfanov 2005, regarding the normalization). From this we get the expectation of $\approx 0.3$ HMXBs brighter than $10^{37} \mathrm{erg} \mathrm{s}^{-1}$, and $\approx 1.2$ sources brighter than $10^{36} \mathrm{erg} \mathrm{s}^{-1}$.

Alternatively we have estimated upper limits for the numbers of HMXBs from the $\mathrm{H} \alpha$ and FIR luminosities reported by Devereux et al. (1994). For $\mathrm{H} \alpha$ the combined luminosity from the nuclear region and from diffuse emission inside the star forming ring (which is at a radius of $\sim 50^{\prime}$, much larger than the maximum distance of $12^{\prime}$ analysed in this paper) is $4.3 \times 10^{39} \mathrm{erg} \mathrm{s}^{-1}$ (corrected to our distance of $780 \mathrm{kpc}$ ). From Grimm et al. (2003) we find that this corresponds to 1.1 HMXBs with a luminosity above $10^{37} \mathrm{erg} \mathrm{s}^{-1}$, and 4.4 HMXBs with a luminosity above $10^{36} \mathrm{erg} \mathrm{s}^{-1}$. The FIR luminosity in this region is $5.25 \times 10^{8} L_{\odot}$, which corresponds to $2.0 \mathrm{HMXBs}$ with a luminosity above $10^{37} \mathrm{erg} \mathrm{s}^{-1}$, and 8.0 HMXBs with a luminosity above $10^{36} \mathrm{erg} \mathrm{s}^{-1}$. It should be noted, however, that the region these luminosities are found from is much larger than the region containing our X-ray data, and that it is very likely that the main part of the light is not produced by star formation, as Devereux et al. (1994) found that for the central region the number of O-type stars is a factor of $\sim 200$ lower than what would be expected if the luminosities were due to star formation. We can therefore safely ignore the contribution of HMXBs in the following analysis.

\subsubsection{Background X-ray sources}

To estimate the number of background sources, we use results of the CXB $\log (N)-\log (S)$ determination by Moretti et al. (2003). We use the source counts in the soft and hard bands (their Eq. (2)) and convert the fluxes to the $0.5-8.0 \mathrm{keV}$ band, assuming a powerlaw spectrum with a photon index of 1.4. For the total area of our survey of $0.126 \mathrm{deg}^{2}$ we obtain from the source counts in the soft band $\sim 1.8 \mathrm{CXB}$ sources above the flux corresponding to $10^{37} \mathrm{erg} \mathrm{s}^{-1}$, and $\sim 29$ above $10^{36} \mathrm{erg} \mathrm{s}^{-1}$.
From the hard band counts the predicted numbers are $\sim 1.2$ and $\sim 30.5$ sources. The predictions based on the soft and hard $\log (N)-\log (S)$ differ slightly because of the well recognized fact that source counts in different energy bands and flux regimes are dominated by different types of sources, see Voss \& Gilfanov (2006). To find the total number of background sources in our source list, we multiply the CXB LF by the incompleteness function found in Sect. 3.3, and integrate over the observed luminosity range. We find the total number of CXB sources to be 89 .

\subsection{The spatial distribution of the point sources}

We studied the azimuthally averaged radial distribution of the X-ray point sources. As the two main contributions of sources are the LMXBs and CXBs, we model the distribution as a superposition of two functions, representing these contributions. As the spatial distribution of the globular clusters in M 31 is significantly different from the mass distribution in the inner parts of the bulge, we have accounted for the globular cluster sources separately. The distribution of LMXBs is assumed to follow the $K$-band light, and for this the image from the 2MASS LGA was used, whereas the density of CXBs can be assumed to be flat on the angular scales under consideration here. The only free parameter of the model is the ratio of normalizations of the LMXB and CXB fraction.

The model was compared to the observations for sources more luminous than $10^{36} \mathrm{erg} \mathrm{s}^{-1}$ (Fig. 1), as sources of such luminosity could be observed in the entire image without it being necessary to consider incompleteness effects. This analysis was presented in Voss \& Gilfanov (2007), where the data was shown to deviate significantly from the model in the inner $r \lesssim 1^{\prime}$. It was also shown that the discrepancy could be succesfully modelled by binaries created through dynamical interactions.

In this paper we adopt the normalizations of the primordial LMXBS and CXBs found in Voss \& Gilfanov (2007). Above 


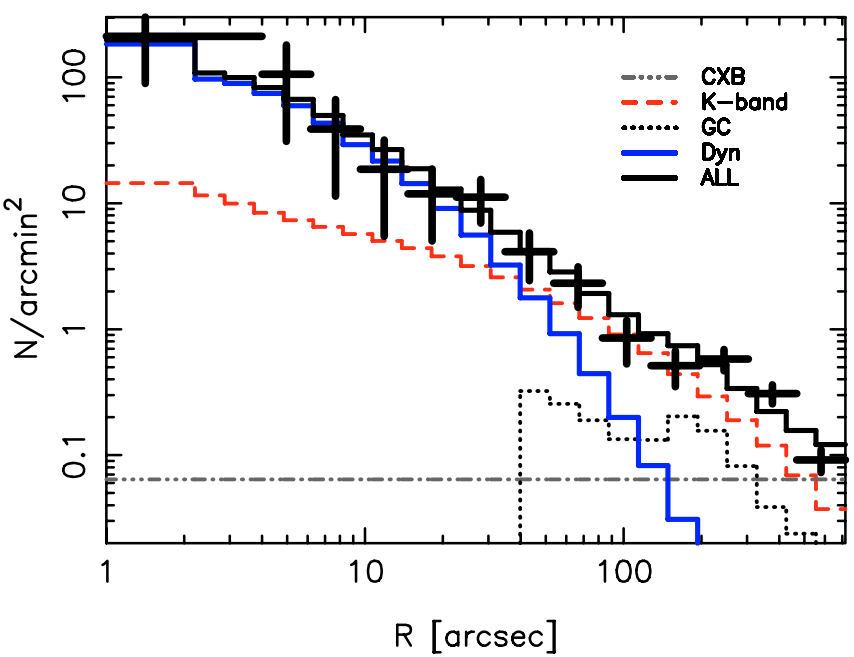

Fig. 1. The spatial distribution of the point sources (crosses), compared to a model consisting of primordial LMXBs, CXB sources and LMXBs in GCs. The GC LMXB normalization was determined from the observed number of matches, and the primordial LMXB and CXB normalizations were found from a fit to the observed source distribution outside $1^{\prime}$. In the inner $1^{\prime}$ the sources follow the expected distribution of LMXBs formed through dynamical interactions, $\rho_{*}^{2}$ (Voss \& Gilfanov 2007).

a luminosity of $10^{36} \mathrm{erg} \mathrm{s}^{-1}$ this corresponds to $29 \mathrm{CXBs}$ and 64 primordial LMXBs, as well as 20 LMXBs created via dynamical interactions in the inner bulge and 20 LMXBs in GCs. The normalization of CXBs is consistent with the expectations. From this we find that the ratio of primordial LMXBs with luminosity above $10^{36} \mathrm{erg} \mathrm{s}^{-1}\left(10^{37} \mathrm{erg} \mathrm{s}^{-1}\right)$ to stellar mass is $N_{x} / M_{*}$ is $17.0 \pm 1.8(8.9 \pm 1.6)$ sources per $10^{10} M_{\odot}$, and the ratio of primordial LMXBs to the $K$-band luminosity is $N_{x} / L_{K}$ is $19.7 \pm 2.1$ (10.3 \pm 1.9 ) sources per $10^{10} L_{\odot, K}$.

The normalization of the primordial LMXBs is about two times smaller than the number obtained by Gilfanov (2004). There are two reasons for this. 1) We removed LMXBs in GCs and in the inner bulge from the analysis, to only account for LMXBs thought to be primordial. This was not done by Gilfanov (2004). 2) We assumed the $K$-band mass to light ratio of the bulge of M 31 to be 0.85 , as compared to the ratio of 0.56 used in Gilfanov (2004).

\subsection{Incompleteness}

The variations of the diffuse background level and deterioration of the PSF at large off-axis angles lead to variations of the point-source sensitivity across the Chandra images. An image in which observations with different pointings are combined has very non-uniform exposure. As a result there are strong incompleteness effects at the faint end of the luminosity function. We calculate the incompleteness function for each analysed area, using the method described in Voss \& Gilfanov (2006), in which the incompleteness function is calculated separately for LMXBs and for CXBs. A completeness limit is calculated for each pixel and weighted by the expected distribution of sources (using the same distributions as in Sect. 3.2). In Fig. 2 the incompleteness function is shown for both LMXBs and CXBs in 3 regions. As in Voss \& Gilfanov (2006) we verified our calculated incompleteness functions by simulations of the type used by Kim \& Fabbiano (2004). In each of the simulations we used Monte Carlo techniques to simulate 10000 point sources. Each

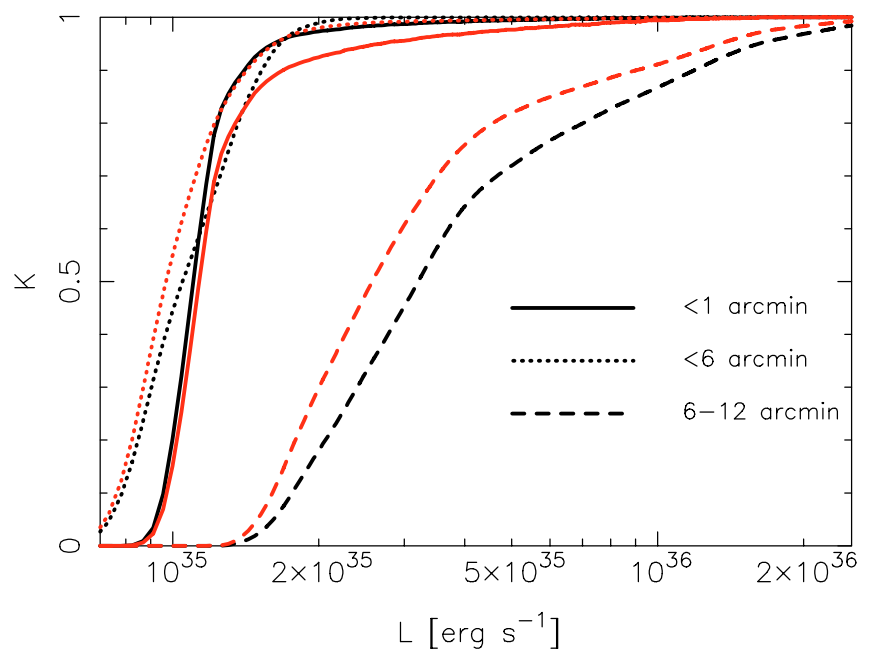

Fig. 2. The incompleteness as a function of source luminosity for 4 regions of the bulge of M31. The red lines show the function for LMXBs, while the black lines show the functions for CXBs.

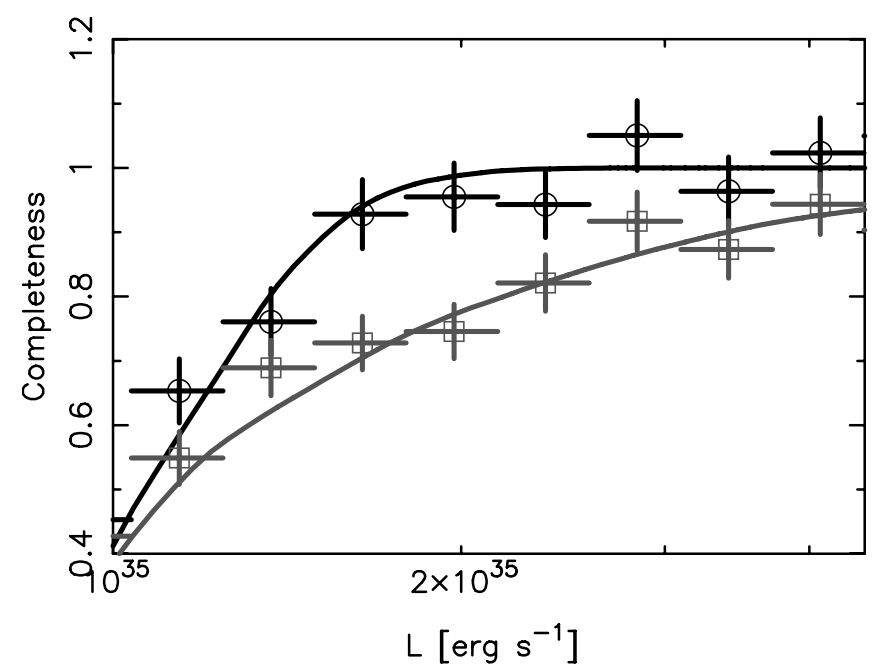

Fig. 3. The results of incompleteness simulations compared to the calculated incompleteness functions of LMXBs. The black line and data points (circles) corresponds to the annulus with $3^{\prime}-6^{\prime}$ distance from the centre of M 31, while the grey line and data points (squares) corresponds to the region within $12^{\prime}$. In both simulations 10000 sources were simulated.

of the sources was placed on the real image of M 31, according to the expected spatial distribution of the source type (LMXBs or CXBs), and our observation pipeline was applied to the image to test if the source is detected, and if so, with what luminosity. This way, the observed number of sources in a luminosity range was compared to the simulated number to determine the detection efficiency. For more details on the method, see Voss $\&$ Gilfanov (2006). The results of the simulations are compared to the calculated functions for two regions in Fig. 3. We calculated the incompleteness function for GCs and GC candidates by finding the detection limit at the position of each of the GCs, and assuming the probability of containing an LMXB to be the same for all the GCs. The incompleteness functions calculated in this section will be used in the analysis of the LF of the LMXBs in Sect. 5. 


\section{Transient sources}

A large fraction of the sources in our sample are variable. For most of the sources, the luminosity varies within a factor of a few. In the combined image the luminosity is the average of the luminosities of the single observations, weighted by the exposure. For the sources with a low amplitude of the variability, this weighted average is adequate for the analysis carried out in this paper.

However, the study of Williams et al. (2006) has shown that, on average, there is $\sim$ one transient source per observation. In an image combined from many observations the effects of these sources on the normalization and shape of the LF are nonnegligible. As the luminosity of a source is weighted by the exposure, it is straightforward that the more observations that are combined, the more transients there are, and the lower the average luminosity of each of them will seem to be. To find and investigate transient sources, we analysed each observation individually in the same way as the combined observation.

For each source it was noted for which observations it was found and with what luminosity. If the source was not found with wavdetect (and if the source region had any exposure) we put the source into one of two categories. The number of photons from the source region, $N_{\mathrm{ps}}$ (85 per cent of PSF centered on the observed coordinates of the full image), and background region $N_{\mathrm{pb}}$ (annulus with radius 1-3 times the source region) were counted. 95 per cent confidence limits $C_{\text {low }}$ and $C_{\text {high }}$ were calculated on the source counts (Gehrels 1986). If $C_{\text {low }}$ was larger than the number of expected background photons in the source region, scaled by exposure and numbers of pixels from the background region, we labelled it a low-significance source and used $N_{\mathrm{ps}}-N_{\mathrm{pb}}$ as source photons to find the luminosity. If $C_{\text {low }}$ was lower than the expectation of background photons, we calculated an upper limit to the luminosity from $C_{\mathrm{high}}-N_{\mathrm{pb}}$. Based on the considerations above we divided the sources into three types for each observation. Type 0 is a source observed with wavdetect, type 1 is a low-significance source, and type 2 is a source not observed, for which an upper limit is given. In Table 3 the source type is given for the observation with lowest luminosity for each source.

The ratio between the highest and the lowest luminosity (or upper limit) was then noted for each of the sources. We chose to label sources with a ratio $>20$ as transients. This gave the 28 sources listed in Table 3. Out of these, only one was actually observed at the lowest luminosity, indicating that the majority of these sources are real transients. For many of our sources, the ratio limit is set so high that if they are transients, they would not be labeled as such, due to lack of exposure. If the limit was set to be lower, however, there would be a large number of sources that are variable, but not transients, that would be included. The amplitude of the variability of a source is artificially enhanced by statistics when the number of observations is large.

A catalogue of transients in M 31 was published by Williams et al. (2006), and 5 further transients were found in a series of papers by the same group (e.g. Williams et al. 2005). From their lists, 36 of the sources are within the region analysed in this paper. Of these, 18 coincide with sources in our transient list. We have therefore identified 10 new sources. Of the 18 sources remaining in their source list, 5 of them were detected in our observations, but did not fulfil our criteria for being transients (they also had high/low ratios lower than 20 in Williams et al. 2006). One source (source 214 in Table 2) was labelled a transient in their paper with a high/low ratio of 96 but only varied by a factor of $<10$ in our observations. The last 12 sources from
Table 3. Highly variable sources with $L_{\max } / L_{\min }>20$. Given here are (1) the source number from Table 1, (2) the ratio of highest observed luminosity to the lowest observed luminosity (or upper limit), (3) the highest observed luminosity, (4) the observation in which the luminosity was highest, (5) the observation in which the luminosity was lowest, and (6) the observational type at lowest luminosity, see description in text. It is also noted in this column if the source belongs to a GC or GC candidate.

\begin{tabular}{|c|c|c|c|c|c|}
\hline $\begin{array}{r}\text { Source } \\
\text { (1) }\end{array}$ & $\begin{array}{r}\text { high/low } \\
\text { (2) }\end{array}$ & $\begin{array}{r}\max \operatorname{lum} \\
\text { (3) }\end{array}$ & $\begin{array}{r}\text { high obs } \\
\text { (4) }\end{array}$ & $\begin{array}{r}\text { low obs } \\
(5)\end{array}$ & $\begin{array}{r}\text { type } \\
(6)\end{array}$ \\
\hline 12 & 22.0 & $7.61 e+36$ & 1585 & 310 & 2 \\
\hline 14 & 468.8 & $7.28 \mathrm{e}+37$ & 308 & 1575 & 2 \\
\hline 23 & 50.3 & $1.26 \mathrm{e}+37$ & 4682 & 305 & 1 \\
\hline 29 & 126.2 & $4.42 \mathrm{e}+37$ & 1575 & 310 & 2 \\
\hline 32 & 65.2 & $1.37 \mathrm{e}+37$ & 4681 & 1575 & 2 \\
\hline 38 & 99.5 & $2.28 \mathrm{e}+37$ & 4679 & 303 & 2 \\
\hline 41 & 28.3 & $3.18 \mathrm{e}+36$ & 303 & 1575 & 1 \\
\hline 44 & 35.7 & $3.22 \mathrm{e}+36$ & 303 & 1575 & $\mathrm{GCC}, 1$ \\
\hline 47 & 64.6 & $1.99 \mathrm{e}+37$ & 4682 & 1575 & 2 \\
\hline 51 & 87.1 & $1.11 \mathrm{e}+37$ & 306 & 1575 & 2 \\
\hline 59 & 589.0 & $4.75 e+37$ & 4682 & 1575 & 2 \\
\hline 68 & 370.5 & $1.93 \mathrm{e}+37$ & 310 & 1575 & 2 \\
\hline 72 & 21.2 & $1.34 \mathrm{e}+37$ & 309 & 4679 & 1 \\
\hline 84 & 54.5 & $7.68 \mathrm{e}+36$ & 1854 & 1575 & 2 \\
\hline 85 & 79.0 & $2.56 \mathrm{e}+37$ & 303 & 1585 & 1 \\
\hline 90 & 58.6 & $5.36 \mathrm{e}+37$ & 311 & 312 & 1 \\
\hline 105 & 954.2 & $3.27 \mathrm{e}+38$ & 1575 & 303 & 2 \\
\hline 118 & 108.4 & $3.86 \mathrm{e}+37$ & 4681 & 303 & 2 \\
\hline 128 & 46.1 & $1.50 \mathrm{e}+37$ & 2896 & 1575 & 2 \\
\hline 130 & 104.4 & $1.49 \mathrm{e}+37$ & 311 & 1575 & 2 \\
\hline 136 & 92.2 & $4.55 \mathrm{e}+37$ & 4682 & 305 & $\mathrm{GCC}, 2$ \\
\hline 146 & 212.4 & $3.77 e+37$ & 4681 & 1575 & 2 \\
\hline 155 & 96.3 & $4.48 \mathrm{e}+37$ & 1585 & 1575 & $\mathrm{GCC}, 0$ \\
\hline 212 & 65.2 & $2.68 \mathrm{e}+37$ & 4682 & 1575 & 2 \\
\hline 216 & 20.8 & $1.04 \mathrm{e}+37$ & 1854 & 1575 & 2 \\
\hline 234 & 66.9 & $1.18 \mathrm{e}+37$ & 4682 & 2898 & 1 \\
\hline 237 & 37.5 & $7.48 \mathrm{e}+37$ & 2897 & 307 & 1 \\
\hline 250 & 53.4 & $7.00 \mathrm{e}+37$ & 2896 & 305 & GC,2 \\
\hline
\end{tabular}

their source list are not active in our observations. Trudolyubov et al. (2006) detected 4 transients with XMM-Newton. 3 of these are not active in our observations, while the fourth (source 234 in Table 2) was found to be transient in our observations, as well as by Williams et al. (2005).

We investigated the spatial distribution of the transient sources. In Fig. 4 we compare their radial distribution with two models: 1 ) the distribution of the $K$-band light, representing the primordial LMXBs, and 2) the distribution of all the observed LMXBs (all sources with CXBs subtracted). It can be seen that with the current set of observations, both models can explain the distribution. With more observations it might be possible to distinguish between the models, and thereby learn if the ratio of transient to persistent sources is different for primordial LMXBs and dynamically formed LMXBs. The number of individual observations and length of these varies strongly from region to region, and this can have significant effects on the observed distribution. Also inside 5" the source density is so high that transients could easily be missed.

\section{The luminosity function of the point sources}

The LF in the bulge of M 31 has previously been studied with Chandra by Kong et al. (2002, 2003); Williams et al. (2004a), but for several reasons it is interesting to do further work on this. The exposure of the inner region has increased significantly 


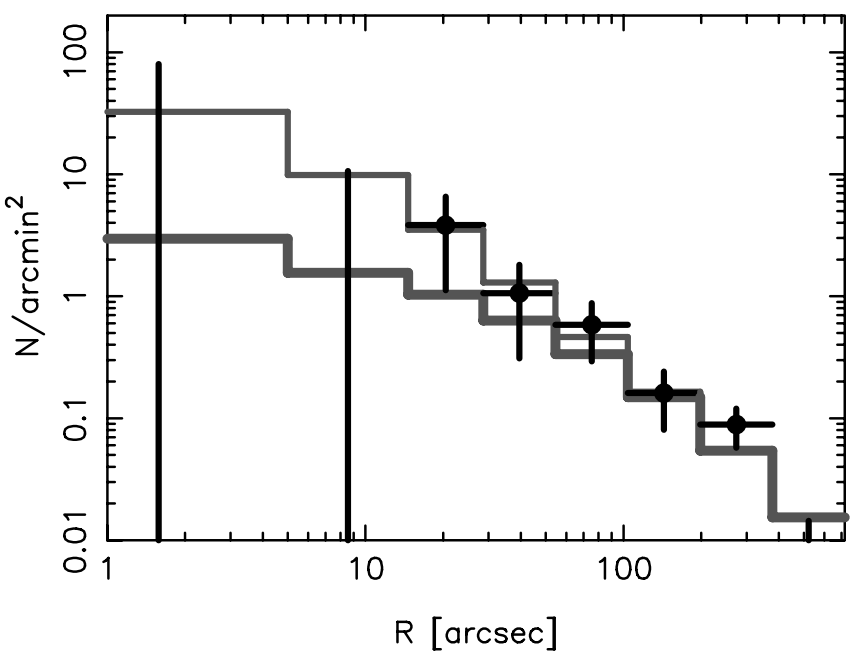

Fig. 4. The spatial distribution of the transient sources, compared to two models, the distribution of primordial LMXBs (thick grey line) and the combined distribution of primordial LMXBs and LMXBs created through dynamical interactions in the inner bulge (thin grey line). The error bars are $1 \sigma$.

since the previous studies, and methods for incompleteness correction have been developed. It is therefore possible to probe the LF at much lower luminosities. Furthermore the previous studies neglected the contribution of background sources. This can be important for the outer parts of the bulge, where the density of LMXBs is comparable to the density of background objects, see Fig. 1.

Here we study the LF of the LMXBs in detail, statistically taking into account the CXBs, and correcting for incompleteness as described in Sect. 3.3. The LMXB LFs presented in this section are corrected by subtracting the LF of CXBs multiplied by the incompleteness function of the CXBs. The normalization of the LF of CXBs was chosen as in 3.2. The LFs of the LMXBs were then corrected by dividing by the incompleteness function of the LMXBs. From Fig. 2 it can be seen that there is a factor of $\sim 10$ difference between the sensitivity in the regions inside $6^{\prime}$ and the regions outside. At the same time the density of X-ray sources is much higher in the inner region than in the outside region, making the $\mathrm{CXB}$ contribution less important. In the inner regions the LF can therefore be determined directly down to a few times $10^{35} \mathrm{erg} \mathrm{s}^{-1}$. We choose to present the functions as differential LFs, as opposed to the cumulative LFs often used in the literature. The advantage of this is that bins are independent, and features in the LF are therefore more visible, and easier to interpret. The disadvantage is that it is necessary to bin the data. The LFs presented below are cut off at a lower luminosity. This luminosity corresponds to the limit at which the incompleteness correction is $>2.5$ for either the CXB LF or the LMXB LF. For this reason the LFs of the individual regions begin at different luminosities in Figs. 5-10. For each of the LFs, we give the number of sources included in the calculations. However it should be noted that due to the corrections for incompleteness and CXB sources applied to the LFs, the error bars in the figures provide better estimates for the precision of the functions.

In Fig. 5 we show the LF of the entire region within a distance of $12^{\prime}$ from the centre of M 31 . The squares correspond to all the sources, whereas to produce the crosses, the transient sources (Table 3 ) were excluded. There is a clear break at $\sim 2 \times 10^{37} \mathrm{erg} \mathrm{s}^{-1}$, consistent with previous results obtained with Chandra (Kong et al. 2002, 2003; Williams et al. 2004a) as well

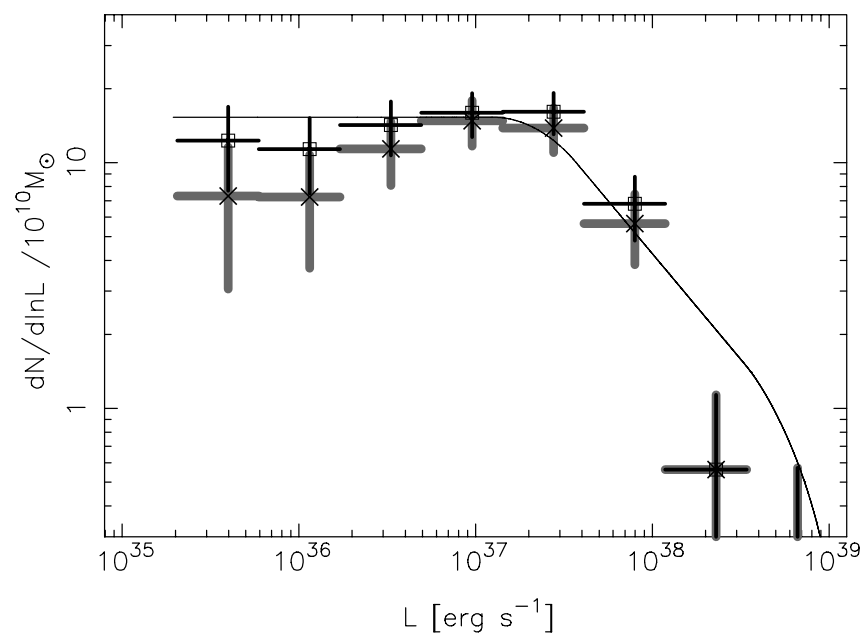

Fig. 5. The LF of LMXBs within $12^{\prime}$ of the centre of M 31. Squares are obtained from all the observed sources (207 sources), whereas for the crosses, the transient sources are excluded (179 sources). Note that in the latter case the relative contribution of sources thought to have dynamical origin is increased, see the discussion in the text. The solid line is the average LF of LMXBs in nearby galaxies from Gilfanov (2004). The normalization is arbitrary, but the same as Figs. 5-10.

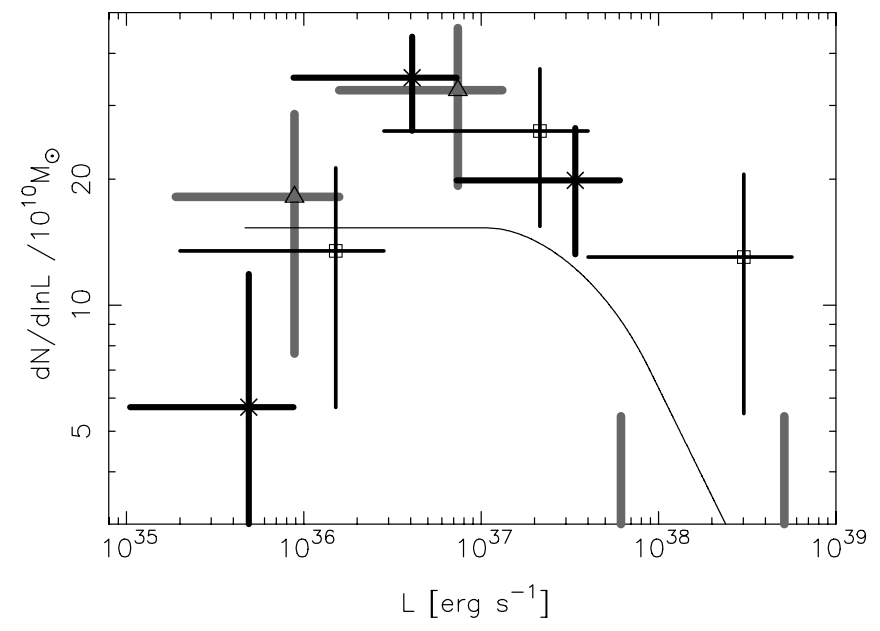

Fig. 6. The LF of the sources in the inner $1^{\prime}$ (crosses, 27 sources), the LF of the X-ray sources in confirmed GCs (squares, 12 sources) and the LF of the X-ray sources in GC candidates (triangles, 9 sources). The normalization of the LFs from GCs and GC candidates is arbitrary. The solid line is the average LMXB LF, with the same normalization in Figs. 5-10.

as other X-ray telescopes (Primini et al. 1993; Shirey et al. 2001; Gilfanov 2004).

As it can be seen in Fig. 5 the effect of transients is to artificially steepen the slope of the LF below $\sim 10^{37} \mathrm{erg} \mathrm{s}^{-1}$. A maximum likelihood (ML) fit by a single powerlaw in the range $2 \times 10^{35}-10^{37} \mathrm{erg} \mathrm{s}^{-1}$ gives a slope of $-0.85 \pm 0.12$ and $-0.7 \pm 0.16$ with and without transients, respectively. While the difference is not statistically significant in our sample, it is a systematic effect that should not be ignored in general when studying a large number of observations combined. To avoid distortion of the LF due to transient sources, they should be excluded from the analysis, and we have done so in the rest of the analyses presented in this paper.

After the transient sources have been excluded, the faint end $\left(2 \times 10^{35}-10^{37} \mathrm{erg} \mathrm{s}^{-1}\right)$ of the LF appears to be significantly flatter than $L^{-1}$ powerlaw. This is caused by the sources located in 


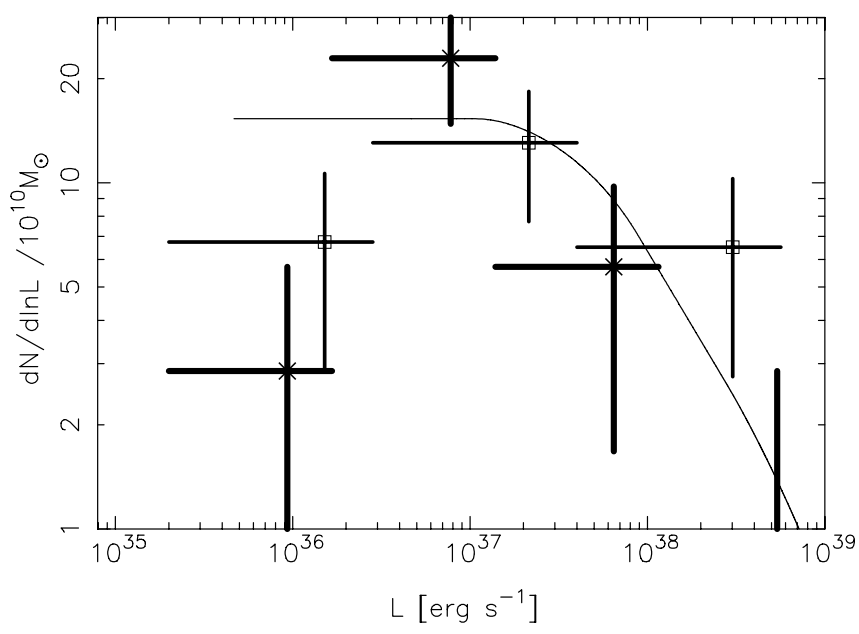

Fig. 7. The LF of the LMXBs in Galactic GCs (crosses, 12 sources) compared to the LF of the LMXBs in confirmed GCs in M 31 (squares, 12 sources). The solid line is the average LMXB LF, with the same normalization in Figs. 5-10.

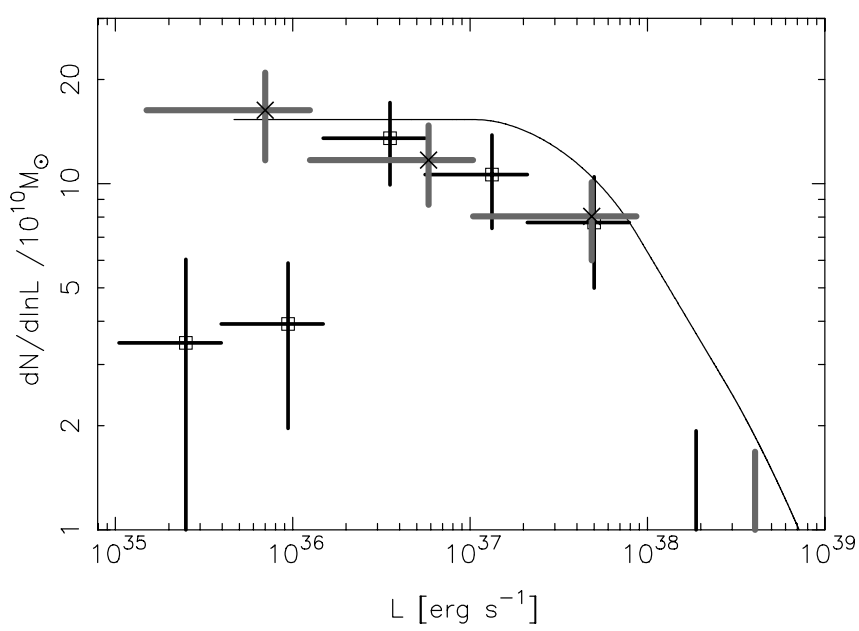

Fig. 8. The LF of sources with a radial distance of $1^{\prime}-9^{\prime}$ from the centre of M31, excluding sources in GCs and GC candidates (crosses, 130 sources), compared to the LF of sources from the inner $1^{\prime}$ and sources in confirmed GCs (squares, 40 sources). The normalization of the latter is arbitrary. The solid line is the average LMXB LF, with the same normalization in Figs. 5-10.

globular clusters and in the inner bulge, thought to have dynamical origin, as the LF of these sources has a prominent fall-off at low luminosities (Fig. 6). Their relative contribution to the LF increases when the transient sources are excluded. As will be shown below, the LF of the primordial sources is consistent with the general shape found by Gilfanov (2004).

In Fig. 6 the LFs of the LMXBs thought to be created through dynamical interactions are presented. The LMXBs from the inner $1^{\prime}$ of the M 31 bulge are shown (crosses), compared to the LFs of LMXBs in confirmed GCs (squares) and GC candidates (triangles). The three LFs are consistent with each other, and for all three populations the number of LMXBs falls off at luminosities below $\log L_{\mathrm{x}} \lesssim 36.0-36.5$. This is most significant in the bulge population, which can also be observed to the lowest luminosity level. For the GC candidate sources, the falling off at low luminosities is hardly significant, but it is known that the GC candidate list is contaminated by background galaxies (Galleti et al. 2004), and with the LF of CXB sources, the

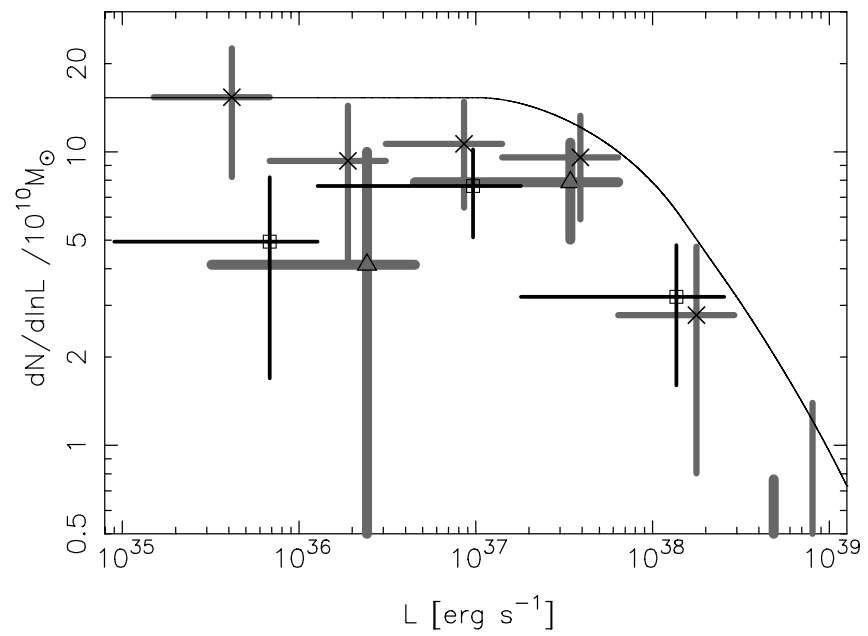

Fig. 9. The LF of sources with a radial distance of $1^{\prime}-3^{\prime}$ (crosses, 28 sources), $3^{\prime}-6^{\prime}$ (squares, 56 sources) and $6^{\prime}-12^{\prime}$ from the centre of M31, excluding sources in GCs and GC candidates (triangles, 58 sources). The solid line is the average LMXB LF, with the same normalization if Figs. 5-10.

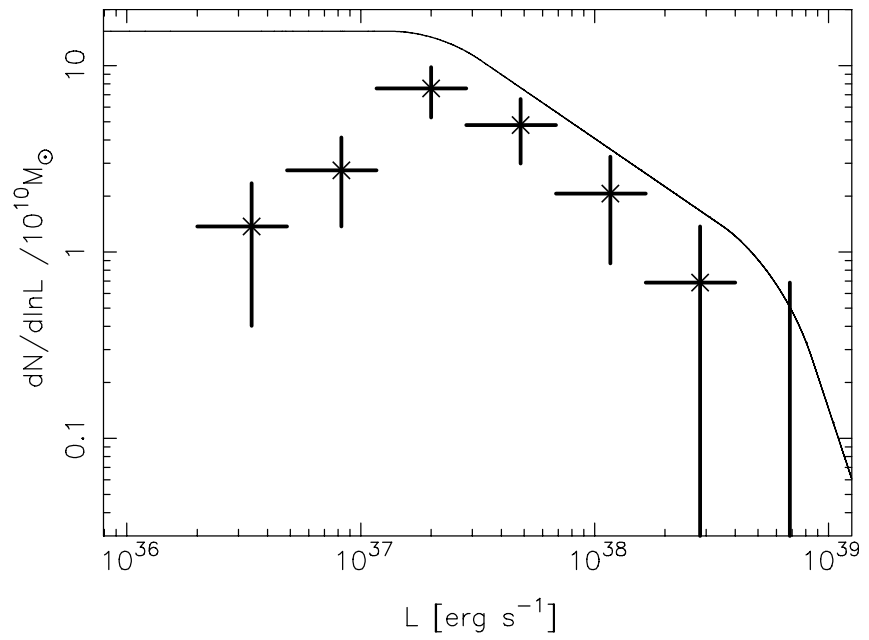

Fig. 10. The LF of the maximum luminosity of the transients observed (28 sources). Note, that below $\sim 10^{37} \mathrm{erg} / \mathrm{s}$ it is subject to strong selection effects. The solid line is the average LMXB LF, with the same normalization in Figs. 5-10.

effect of such a contamination would be to raise the lower end of the LF.

In the inner $1^{\prime}$ of M31, as well as in the GCs, the source density is so high that source blending can become a factor. We performed Monte Carlo simulations of the source population in the inner $1^{\prime}$ of M31, similar to the ones performed to estimate incompleteness (see Sect. 3.3). We assumed the average luminosity function of Gilfanov (2004), with the normalization according to our observed number of sources in the region, and with a lower cut-off at $10^{36} \mathrm{erg} \mathrm{s}^{-1}$ as observed, and the spatial distribution of all sources in Fig. 1. From this we find that the fraction of blended sources (parameter $b$ in Appendix A) is $\sim 3-4 \%$. For an alternative luminosity function in which the lower cut-off is set at $10^{34} \mathrm{erg} \mathrm{s}^{-1}, b \sim 9-10 \%$.

As only $\sim 20 \%$ of the GCs host LMXBs, the fraction of blended sources is also likely to be low here $(b \sim 4$ per cent, assuming that all GCs are identical, but the exact number depends on the distribution of GC properties relevant for the formation of LMXBs). For comparison one out of $12 \mathrm{GCs}$ 
hosting LMXBs in the Galaxy has been shown to host two LMXBs (White \& Angelini 2001), corresponding to $b \sim 8-9$ per cent. In Appendix A we consider the effects of source blending on the observed LF, and we show that for the values of $b$ in this range, the effect of blending is not important.

Given the Chandra angular resolution, at the distance of M 31, all X-ray sources in a GC will be blended into one pointlike source. As there are numerous sources of low luminosity $L_{X} \lesssim 10^{34} \mathrm{erg} \mathrm{s}^{-1}$, this could affect our analysis. This is not the case, however, as the luminosities of these sources are too low. For example the combined luminosity of the $\sim 300 \mathrm{ob}-$ served sources in the massive Galactic GC 47 Tucanae is $\sim 5 \times$ $10^{33} \mathrm{erg} \mathrm{s}^{-1}$ (Heinke et al. 2005), i.e. less than $1 \%$ of the luminosity of a typical GC source observed in M 31.

From a comparison of Figs. 5 and 6, it appears that the LF of the LMXBs of presumably dynamical origin is different from the average LF of all the LMXBs. We investigate this difference further in Fig. 8 where we compare the LF of the dynamically formed LMXBs (sources located in the inner $1^{\prime}$ and in confirmed GCs) with the LF of all other sources in the $1^{\prime}-9^{\prime}$ annulus. These are, presumably, of primordial origin. This figure confirms qualitatively the difference between the two populations. Due to rather limited numbers of sources the LFs are not very tightly constrained. In particular, the statistics is insufficient to discriminate between a genuine low-luminosity cut-off in the LF of dynamically formed sources and its moderate flattening. To estimate the statistical significance we consider the numbers of sources in different sub-populations in the $1.5 \times 10^{35}-10^{36} \mathrm{erg} \mathrm{s}^{-1}$ luminosity range (the lower boundary is defined by the low bound of the primordial LF, see discussion earlier in this section). There are two sources in this luminosity range in the population of the dynamically formed LMXBs, whereas $17.4 \pm 4$ would be expected if the source counts in the $10^{36}-10^{37} \mathrm{erg} \mathrm{s}^{-1}$ range were extrapolated with a $\mathrm{d} N / \mathrm{d} L \propto L^{-1}$ law. Due to nearly identical normalizations of the two LFs above $\log \left(L_{\mathrm{X}}\right) \gtrsim 36$ (cf. Fig. 8), these numbers can be directly compared with $32 \pm 11$ primordial sources (CXB contribution subtracted and incompleteness corrected) observed in the same luminosity range $(20 \pm$ 5.6 sources expected for $L^{-1}$ extrapolation). In order to further quantify the difference between the two luminosity distributions we fit them with a single powerlaw in the $1.5 \times 10^{35}-10^{37} \mathrm{erg} \mathrm{s}^{-1}$ luminosity range, using ML fits. For the primordial sources we obtained a differential slope of $-1.11 \pm 0.18$, while the LF of dynamicaly formed LMXBs has a slope of $-0.6 \pm 0.2$. Although the difference between these two numbers is only marginally significant, the LF slope of the dynamically formed LMXBs is inconsistent with the value of -1 obtained for the average LMXB LF.

It is interesting to compare the results with the bright $\left(L_{\mathrm{x}} \gtrsim\right.$ $10^{35} \mathrm{erg} \mathrm{s}^{-1}$ ) LMXBs in Galactic GCs. Currently 13 of such LMXBs have been observed in $12 \mathrm{GCs}$, and due to the proximity the sample is believed to be complete, except for possible future transients. While all of these sources have been observed with Chandra, there are not published luminosities for all of them, and analysis of the observations are beyond the scope of this paper. Instead we find the luminosities by averaging the lightcurves for each of the sources from RXTE ASM, over all of the observed time (until January 1st, 2007). The count rates were converted to fluxes in the $0.5-8.0 \mathrm{keV}$ band, assuming a powerlaw spectrum with photon index 1.7 , using PIMMS ${ }^{2}$. This gives a conversion

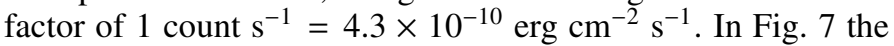
LF of the LMXBs in Galactic GCs is compared to the LF of

\footnotetext{
${ }^{2}$ http://cxc.harvard.edu/toolkit/pimms.jsp
}

LMXBs in confirmed GCs in M 31, and it is shown that in the Galaxy there is a clear cut-off at $\sim 10^{36} \mathrm{erg} \mathrm{s}^{-1}$.

The difference between the LF of primordial and dynamically formed LMXBs is interesting for several reasons. It has recently been discussed whether most of the field LMXBs were actually formed in GCs (e.g. White et al. 2002; Juett 2005). If the LFs of the field and GC LMXBs are different, it is a strong indication that their origin is different as well. Moreover, the fact that the LF of the sources in the inner $1^{\prime}$ is consistent with the LF of the GC sources and different from the LF of the field LMXBs reinforces the conclusion of Voss \& Gilfanov (2007) that the surplus X-ray sources in the center of M 31 are LMXBs created through dynamical interactions in high stellar density environment of the inner bulge.

Differences between the LFs of dynamically formed LMXBs in the globular clusters and field LMXBs have previously been reported (Kong et al. 2002; Trudolyubov \& Priedhorsky 2004) but at larger luminosities, above $10^{37} \mathrm{erg} \mathrm{s}^{-1}$. These have been disputed in a recent thorough study of six elliptical galaxies by Kim, E. et al. (2006), who concluded that the LFs of the two populations are consistent. We note that the differences found in the analysis of this paper occur at luminosities below the sensitivity threshold of Kim, E. et al. (2006), and our results therefore do not contradict theirs.

Various models for LMXB evolution exist, from which the shape of their luminosity distribution can be predicted. For a population of LMXBs with non-degenerate donors, the differential powerlaw slope of -1 at luminosities below $\sim 10^{37}$ can be naturally obtained if the mass transfer is driven by gravitational radiation, as opposed to the steeper slope above $\sim 10^{37} \mathrm{erg} \mathrm{s}^{-1}$, which can be explained by the magnetic braking driven systems (Postnov \& Kuranov 2005; Pfahl et al. 2003). Ultra-compact $\mathrm{X}$-ray binaries (UCXB) have degenerate donor stars and the mass transfer is driven by gravitational radiation alone. In this case the reaction of the WD donor to mass loss is important for the mass transfer rates and therefore also for slope of the LF, and models have been succesful in explaining the bright end of the LMXB LF, near and above $\sim 10^{38} \mathrm{erg} \mathrm{s}^{-1}$ (Bildsten \& Deloye 2004). No modeling of the fainter end of luminosity distribution for the UCXB population has been reported so far. Intuitively, one might expect that the luminosity distribution of these systems should fall off at low luminosities. Although the UCXB systems are very unlikely to contribute significantly to the bulk of fainter primordial LMXBs in the $\log \left(L_{\mathrm{X}}\right) \lesssim 37$ luminosity domain, their importance increases dramatically in the entire luminosity range when considering the LMXBs of dynamical origin, especially those formed in the high velocity environment of the inner bulge (Voss \& Gilfanov 2007). This offers a plausible explanation for the rather peculiar shape of the luminosity distribution of the globular cluster sources and of the sources in the inner $1^{\prime}$ of M31. As the reaction of the WD donor to mass loss depends on the chemical composition of the WD, modeling the luminosity function at low luminosities and comparing with observations of LMXB in the inner bulge and in globular clusters in M 31 and other galaxies might reveal new information on the progenitors of the UCXBs and advance our understanding of binary evolution and dynamical interactions in dense stellar environments in general.

Another factor, potentially important at low mass transfer rates, is the thermal-viscous instability, which causes transient behaviour in LMXBs below some critical value of the mass accretion rate (van Paradijs 1996). Consequently, the LF of persistent sources should be expected to have a break around 
this luminosity. The critical luminosity is somewhere in the $\$ 10^{35-36} \mathrm{erg} \mathrm{s}^{-1}$ domain, and depends, among other parameters, on the physical size of the accretion disk around the compact object (King \& Ritter 1998). In this picture, if the disk instability was the reason for the observed low luminosity cut-off observed in Fig. 6, the critical luminosity for UCXBs would be expected to be lower than for LMXBs with non-degenerate donors. This prediction seems to be in contrast to the result of this paper, that the LF of the dynamically formed LMXBs (presumably having a significantly higher fraction of UCXBs) appears to be flatter (i.e. fewer faint systems) than the LF of the primordial LMXBs.

In the previous study of LMXBs in the bulge of M 31 (Kong et al. 2002), it was found that their LF varied significantly with the distance from the centre, becoming progressively steeper with radius. We searched for the radial trend by comparing the LFs of the primordial LMXBs (that is, with LMXBs in GCs excluded) of the annuli $1^{\prime}-3^{\prime}, 3^{\prime}-6^{\prime}$ and $6^{\prime}-12^{\prime}$ (Fig. 9), and found no statistically significant variations. We suggest that the difference in the LF reported by them, especially between their regions 2 and 3, was caused by the contribution of CXB sources which becomes more larger in the outer parts of the bulge (cf. Fig. 1). Note that this possibility was also considered by Kong et al. (2002).

In Fig. 10, we show the LF of transient sources. As the average luminosity is meaningless, due to its dependence on the exposure time and pattern of the observations in which the sources were found, we have chosen to use the maximum of the observed luminosities in the individual observations for each source. The LF of these sources follows the average LF of LMXBs to a minimum luminosity of $10^{37} \mathrm{erg} \mathrm{s}^{-1}$. Below this the observed distribution falls off quickly, but this is likely an artifact of the our selection criterion for transient sources, $F_{\max } / F_{\min }>20$. For most sources with peak luminosities below $10^{37} \mathrm{erg} \mathrm{s}^{-1}$, it is not possible to constrain the quiescent luminosity well enough to classify the sources as transients.

\section{Conclusions}

We have studied the X-ray point sources in the bulge of M 31 $\left(r<12^{\prime}\right)$ based on archival Chandra data. Our study concentrated on statistical properties of the population, with emphasis on the spatial distribution and LFs of the various subpopulations. One of our primary goals was to contrast properties of different sub-populations of X-ray point sources, namely transient and persistent sources, primordial LMXBs and dynamically formed ones. To achieve this we combined 26 Chandra observations to obtain as much exposure as was possible and implemented an adequate correction for incompleteness effects, as well as for contamination from background sources.

With a total exposure time of $201 \mathrm{ks}$, we detected $263 \mathrm{X}$-ray point sources within a distance of $12^{\prime}$ from the centre of M31. Of these sources 64 were not observed previously. This allowed us to study the sources to a minimum luminosity of $\sim 10^{35} \mathrm{erg} \mathrm{s}^{-1}$, whereas the sample is complete above $\sim 10^{36} \mathrm{erg} \mathrm{s}^{-1}$. We found good agreement between the observed number of sources and the expected number, predicted based on the $K$-band luminosity and average $\mathrm{X}$-ray mass to light ratio for nearby galaxies. The radial distribution of the M31 sources (Fig. 1) can be interpreted as superposition of the following three components: (i) primordial LMXBs following the $K$-band light profile, (ii) LMXBs created through dynamical interactions in the inner bulge of the galaxy, with a distribution that follows the square of the stellar density $\rho_{*}^{2}$ and (iii) LMXBs dynamically created in the globular clusters, with a radial profile that follows the distribution of globular clusters in M 31. Superimposed on these are the CXB sources, the distribution of which is flat on the angular scales under consideration.

After applying the incompleteness correction and subtracting the contribution of $\mathrm{CXB}$ sources, we were able to recover the LF of M31 sources down to the luminosity of $\sim$ few $\times$ $10^{35} \mathrm{erg} \mathrm{s}^{-1}$, which is a factor of $\sim 3$ better than previous studies. The luminosity distribution of all X-ray sources in the bulge of M 31 (Fig. 5) is consistent with the average LMXB LF obtained by Gilfanov (2004), in particular, it follows the $\mathrm{d} N / \mathrm{d} L \propto L^{-1}$ law in the faint luminosity limit, in agreement with the behaviour found earlier for LMXBs in the Milky Way and in Cen A. It was furthermore possible to divide the LMXBs into two subpopulations - primordial LMXBs and dynamically formed ones, in order to study the differences in their luminosity distributions (Fig. 8). We found that the LF of the primordial LMXBs is consistent with the average LMXB LF, and is independent of the radial distance from the centre of M31, within the accuracy allowed by the statistics of our sample. The LMXBs thought to be of dynamical origin have a significantly different luminosity distribution - below $\log \left(L_{X}\right) \lessgtr 36.5$ their LF shows a prominent decrease towards low luminosities (Fig. 6). The statistics is not sufficient to tightly constrain the shape of the LF, in particular to distinguish between a true low luminosity cut-off and a more moderate flattening of the luminosity function. It is however sufficient to claim that the low luminosity, $\log \left(L_{X}\right)<37$, slope of the LF of these sources, $-0.6 \pm 0.2$, is inconsistent with the $\mathrm{d} N / \mathrm{d} L \propto L^{-1}$ law.

We identified the population of transient sources and found that their radial distribution is consistent with the distribution of persistent sources. However the current statistics is insufficient to investigate differences between the fractions of transients in primordial and dynamically formed LMXBs, especially in the inner $30^{\prime \prime}$, where the radial distribution of these two populations differ the most. Above $\sim 10^{37} \mathrm{erg} \mathrm{s}^{-1}$ the LF of the maximum luminosity of the transients follows the average LMXB LF (Fig. 10).

Acknowledgements. This reaserch has made use of CHANDRA archival data provided by the CHANDRA X-ray Center and data from the 2MASS Large Galaxy Atlas provided by NASA/IPAC infrared science archive, as well as $A S M / R X T E$ data obtained through the HEASARC online service. We thank the referee for helpful remarks on the paper.

\section{Appendix A: The effects of source blending on the luminosity function}

In densely populated regions of X-ray point sources, such as GCs or the very inner parts of a galaxy, a fraction of the observed point sources will be a blend of two or more sources. Here we present a brief investigation of the effects of such source blending on the observed LF. If the distribution of luminosities of single sources is given by $P_{1}(L)$, then the distribution of luminosities $P_{2}(L)$ of a blend of two sources is given by

$P_{2}(L)=\int_{0}^{L} P_{1}(\xi) P_{1}(L-\xi) \mathrm{d} \xi$.

Ignoring blends of three or more sources, the observed luminosity distribution is then given by

$P_{\mathrm{d}}(L)=(1-b) P_{1}(L)+b P_{2}(L)$

where $b$ is the fraction of observed sources that are blends of two sources. In Fig. A.1 we show the effects of source blending on the LMXB LF of Gilfanov (2004). The two models shown have 


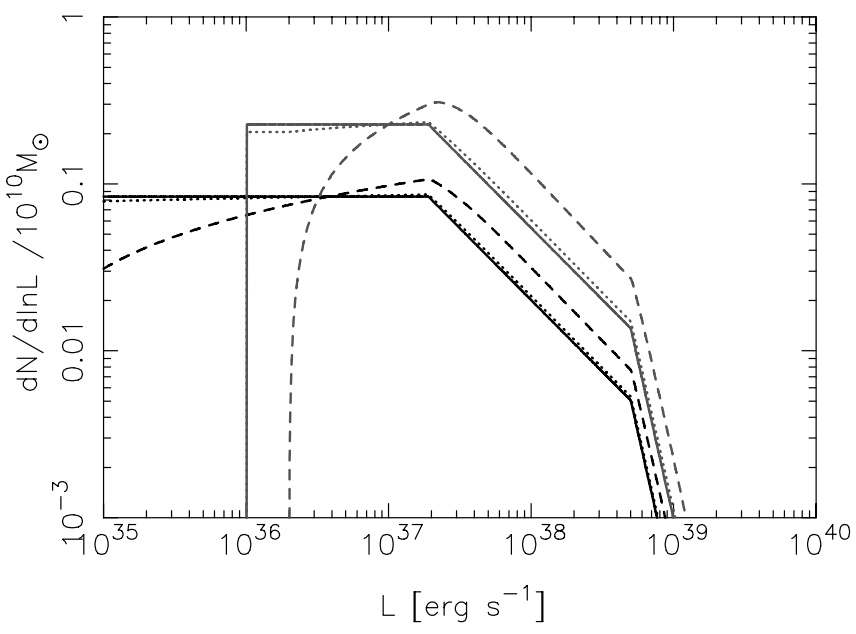

Fig. A.1. The effects of blending on the LMXB LF of Gilfanov (2004). The solid lines are the LFs without blending, whereas the dashed and dotted lines are the LFs assuming $b=1$ and $b=0.1$, respectively.

different lower cut-off luminosities, below which the functions are set to zero, $10^{34} \mathrm{erg} \mathrm{s}^{-1}$ for the lower model and $10^{36} \mathrm{erg} \mathrm{s}^{-1}$ for the upper model (the assumed LFs are therefore equal to the LFs for which $b$ was calculated in Sect. 5). The solid lines give $P_{1}(L)$, whereas the dashed lines gives $P_{2}(L)$. The dotted lines gives $P_{\mathrm{d}}(L)$, for $b=0.10$. It is clear that for sources samples with $b \lesssim 0.10$ the effects on the observed LF are negligible, and even for samples with higher values of $b$, the effects are relatively small.

\section{References}

Bell, E., \& De Jong, R. 2001, ApJ, 550, 212

Bildsten, L., \& Deloye, C. J. 2004, ApJ, 607, L119

Börngen, F. 1968, Astron. Nachr., 291, 19

Braun, R., AJS, 72, 761

Ciardullo, R., Ford, H. C., Neill, J. D., Jacoby, G. H., \& Shafter, A. W. 1987, ApJ, 318, 520

Ciardullo, R., Jacoby, G. H., Ford, H. C., \& Neill, J. D. 1989, ApJ, 339, 53

Crampton, D., Cowley, A. P., Hutchings, J. B., Schade, D. J., \& van Speybroeck, L. P. 1984, ApJ, 284, 663

Deloye, C. J., \& Bildsten, L. 2003, ApJ, 598, 1217

Devereux, N. A., Price, R., Wells, L. A., \& Duric, N. 1994, AJ, 108, 1667

Dickey, J. M., \& Lockman, F. J. 1990, ARA\&A, 28, 215

Fabbiano, G. 2006, ARA\&A, 44, 323
Fan, Z., Ma, J., Zhou, X., Chen, J., Jiang, Z., \& Wu, Z. 2005, PASP, 117, 1236 Galleti, S., Federici, L., Bellazzini, M., Fusi Pecci, F., \& Macrina, S. 2004, A\&A, 416, 917

Gehrels, N. 1986, ApJ 303, 336

Gelfand, J. D., Lazio, T. J. W., \& Gaensler, B. M. 2004, ApJS, 155, 89 Gilfanov, M. 2004, MNRAS, 349, 146

Grimm, H.-J., Gilfanov, M. R., \& Sunyaev, R. A. 2003, MNRAS, 339, 793

Harnden, F. R., Fabricant, D. G., Harris, D. E., \& Schwarz, J. 1984, SAO Special Report \#393

Heinke, C. O., Grindlay, J. E., Edmonds, P. D., et al. 2005, ApJ, 625, 796

Jarret, T. H., Chester, T., Cutri, R., Schneider, S., \& Huchra, J. P. 2003, AJ, 125, 525

Juett, A. M. 2005, ApJ, 621, L25

Kaaret, P. 2002, ApJ, 578, 114

Kim, D.-W., \& Fabbiano, G. 2004, ApJ, 611, 846

Kim, D.-W., Fabbiano, G., Kalogera, et al. 2006 [arXiv: astro-ph/0606008]

Kim, E., Kim, D.-W., Fabbiano, G., et al. 2006, ApJ, 647, 276

King, A. R., \& Ritter, H. 1998, MNRAS, 293, L42

Kong, A. K. H., Garcia, M. R., Primini, F. A., et al. 2002, ApJ, 577, 738

Kong, A. K. H., Di Stefano, R., Garcia, M. R., \& Greiner, J. 2003, ApJ, 585, 298

Macri, L. M. 2001, ApJ, 549, 721

Magnier, E. A. 1993, Ph.D. Thesis, MIT

Magnier, E. A., Prins, S., van Paradijs, J., et al. 1995, A\&AS, 114, 215

Meyssonnier, N., Lequeux, J., \& Azzopardi, M. 1993, A\&AS, 102, 251

Monet, D. G., Levine, S. E., Canzian, B., et al. 2003, AJ, 125, 984

Moretti, A., Campana, S., Lazzati, D., \& Tagliaferri, G. 2003, ApJ, 588, 696

Morrison, J. E., Röser, S., McLean, B., Bucciarelli, B., \& Lasker, B. 2001, AJ, 121,1752

Pfahl, E., Rappaport, S., \& Podsiadlowski, P. 2003, ApJ, 597, 1036

Pietsch, W., Fliri, J., Freyberg, M. J., et al. 2005a, A\&A, 442, 879

Pietsch, W., Freyberg, M. J., \& Haberl, F. 2005b, A\&A, 434, 483

Postnov, K. A., \& Kuranov, A. G. 2005, Astr. Lett., 31, 7

Primini, F. A., Forman, W., \& Jones, C. 1993, ApJ, 410, 615

Shafter, A. W., \& Irby, B. K. 2001, ApJ, 563, 749

Shirey, R., Soria, R., Borozdin, K., et al. 2001, A\&A, 365, 195

Shtykovskiy, P., \& Gilfanov, M. 2005, A\&A, 431, 597

Skrutskie, M. F., Cutri, R. M., Stiening, R., et al. 2006, AJ, 131, 1163

Stanek, K. Z., \& Garnavich, P. M. 1998, ApJ, 503, 131

Trudolyubov, S., \& Priedhorsky, W. 2004, ApJ, 616, 821

Trudolyubov, S., Priedhorsky, W., \& Cordova, F. 2006, ApJ, 645, 277

van Paradijs, J. 1996, ApJ, 464, L139

Voss, R., \& Gilfanov, M. 2006, A\&A, 447, 71

Voss, R., \& Gilfanov, M. 2007, in preparation

Walterbos, R. A. M., \& Kennicutt, R. C. 1987, A\&AS, 69, 311

White, N. E., \& Angelini, L. 2001, ApJ, 561, L101

White, R. E., Sarazin, C. L., \& Kulkarni, S. R. 2002, ApJ, 504, L31

Williams, B. F. 2003, AJ, 126, 1312

Williams, B. F., Garcia, M. R., Kong, A. K. H., et al. 2004a, ApJ, 609, 735

Williams, B. F., Garcia, M. R., McClintock, J. E., \& Kong, A. K. H. 2004b, AJ, 128,1588

Williams, B. F., Garcia, McClintock, J. E., Primini, F. A., \& Murray, S. S. 2005, ApJ, 632, 1086

Williams, B. F., Naik, S., Garcia, M. R., \& Callanan, P. J. 2006, ApJ, 643, 356 
R. Voss and M. Gilfanov: LF of X-ray point sources in the bulge of M31, Online Material p 1

\section{Online Material}


R. Voss and M. Gilfanov: LF of X-ray point sources in the bulge of M31, Online Material p 2

Table 2. The list of point like X-ray sources within a distance of $12^{\prime}$ from the centre of M 31 .

\begin{tabular}{|c|c|c|c|c|c|c|c|c|c|c|}
\hline $\begin{array}{c}\text { Number } \\
\text { (1) }\end{array}$ & $\begin{array}{l}\text { distance } \\
\text { (2) }\end{array}$ & $\begin{array}{l}\text { RA } \\
(3)\end{array}$ & $\begin{array}{l}\text { Dec } \\
(4)\end{array}$ & $\begin{array}{l}\text { cts } \\
(5)\end{array}$ & $\begin{array}{r}\text { corrected cts } \\
\text { (6) }\end{array}$ & $\begin{array}{r}\text { error } \\
(7)\end{array}$ & $\begin{array}{c}\text { luminosity } \\
\text { (8) }\end{array}$ & $\begin{array}{l}\text { type } \\
\text { (9) }\end{array}$ & $\begin{array}{l}\text { id } \\
\text { (10) }\end{array}$ & $\begin{array}{c}\text { name } \\
(11)\end{array}$ \\
\hline 1 & 1.0 & 004244.37 & 411608.7 & 2245 & 2580.7 & 58.0 & $7.08 \mathrm{e}+36$ & & & $\mathrm{r} 1-10$ \\
\hline 2 & 2.2 & 004244.38 & 411607.4 & 2864 & 3308.2 & 65.4 & $1.08 \mathrm{e}+37$ & & & r1-9 \\
\hline 3 & 4.0 & 004244.38 & 411605.4 & 1117 & 1233.9 & 41.3 & $5.32 \mathrm{e}+36$ & & & r1-21 \\
\hline 4 & 4.6 & 004244.30 & 411614.0 & 316 & 344.9 & 22.6 & $1.52 \mathrm{e}+36$ & & & r1-22 \\
\hline 5 & 5.4 & 004243.86 & 411611.1 & 225 & 244.1 & 19.2 & $1.07 e+36$ & & & r1-27 \\
\hline 6 & 7.4 & 004243.87 & 411603.9 & 709 & 811.6 & 33.1 & $3.53 e+36$ & & & r1-23 \\
\hline 7 & 9.7 & 004244.68 & 411618.2 & 906 & 1047.3 & 37.3 & $4.62 \mathrm{e}+36$ & & & $\mathrm{r} 1-8$ \\
\hline 8 & 10.6 & 004245.24 & 411611.1 & 456 & 516.2 & 26.8 & $2.25 \mathrm{e}+36$ & & & r1-20 \\
\hline 9 & 14.5 & 004245.60 & 411608.6 & 1026 & 1191.0 & 39.5 & $5.21 \mathrm{e}+36$ & & & $\mathrm{r} 1-7$ \\
\hline 10 & 15.3 & 004245.12 & 411621.7 & 3098 & 3631.2 & 67.9 & $1.61 \mathrm{e}+37$ & RAD & SIM WSTB 37W135 & r1-4 \\
\hline 11 & 20.8 & 004243.88 & 411629.6 & 1293 & 1463.5 & 44.5 & $6.54 e+36$ & & & r1-11 \\
\hline 12 & 21.7 & 004246.01 & 411619.6 & 468 & 531.3 & 27.2 & $2.33 e+36$ & $\mathrm{t}$ & & r1-19 \\
\hline 13 & 23.8 & 004243.75 & 411632.4 & 2329 & 2726.7 & 59.0 & $1.24 \mathrm{e}+37$ & & & r1-12 \\
\hline 14 & 24.0 & 004242.18 & 411608.3 & 4525 & 5353.9 & 81.6 & $2.45 e+37$ & $\mathrm{t}$ & & $\mathrm{r} 1-5$ \\
\hline 15 & 25.9 & 004242.48 & 411553.7 & 3077 & 3601.1 & 67.6 & $1.62 \mathrm{e}+37$ & PN & CIA 4 & r1-14 \\
\hline 16 & 30.1 & 004243.00 & 411543.2 & 2424 & 2839.4 & 60.1 & $1.29 \mathrm{e}+37$ & & & r1-13 \\
\hline 17 & 30.6 & 004246.97 & 411615.6 & 4037 & 4736.5 & 77.3 & $2.07 e+37$ & & & $\mathrm{r} 1-3$ \\
\hline 18 & 33.3 & 004243.20 & 411640.3 & 413 & 473.2 & 25.6 & $2.20 \mathrm{e}+36$ & & & r1-24 \\
\hline 19 & 33.5 & 004246.16 & 411543.2 & 372 & 427.0 & 24.3 & $1.91 \mathrm{e}+36$ & & & r1-18 \\
\hline 20 & 37.5 & 004247.17 & 411628.4 & 10653 & 12498.5 & 124.6 & $5.59 \mathrm{e}+37$ & PN & CIA 11 & $\mathrm{r} 1-2$ \\
\hline 21 & 41.0 & 004242.65 & 411645.9 & 38 & 33.6 & 8.8 & $1.58 \mathrm{e}+35$ & & & $\mathrm{X}$ \\
\hline 22 & 42.4 & 004247.87 & 411622.9 & 370 & 425.5 & 24.2 & $1.92 \mathrm{e}+36$ & & & r1-17 \\
\hline 23 & 44.8 & 004247.88 & 411549.8 & 264 & 304.3 & 20.7 & $1.40 \mathrm{e}+36$ & $\mathrm{t}$ & & r1-25 \\
\hline 24 & 45.0 & 004242.08 & 411532.0 & 366 & 417.4 & 24.2 & $2.14 \mathrm{e}+36$ & & & r1-31 \\
\hline 25 & 45.1 & 004245.54 & 411652.3 & 27 & 23.4 & 7.6 & $1.09 e+35$ & & & X \\
\hline 26 & 47.0 & 004245.09 & 411523.2 & 386 & 441.1 & 24.8 & $2.05 e+36$ & PN & CIA 18 & r1-26 \\
\hline 27 & 52.0 & 004248.72 & 411624.5 & 523 & 606.0 & 28.6 & $2.72 \mathrm{e}+36$ & & & r1-16 \\
\hline 28 & 53.3 & 004240.00 & 411547.5 & 4068 & 4772.3 & 77.5 & $2.32 \mathrm{e}+37$ & RNova & SI 1997-06 & r1-15 \\
\hline 29 & 53.5 & 004239.59 & 411614.3 & 2967 & 3500.0 & 66.4 & $1.73 e+37$ & $\mathrm{t}$ & & r1-34 \\
\hline 30 & 53.9 & 004242.53 & 411659.4 & 210 & 240.8 & 18.6 & $1.11 \mathrm{e}+36$ & & & r1-30 \\
\hline 31 & 54.5 & 004247.90 & 411532.9 & 3265 & 3834.7 & 69.6 & $1.84 \mathrm{e}+37$ & & & r1-6 \\
\hline 32 & 55.7 & 004243.79 & 411514.1 & 110 & 117.5 & 13.8 & $5.69 e+35$ & $\mathrm{t}$ & & r1-28 \\
\hline 33 & 55.8 & 004241.45 & 411523.8 & 471 & 540.7 & 27.2 & $2.93 e+36$ & $\mathrm{GC}$ & Bol B124 & r1-32 \\
\hline 34 & 64.7 & 004238.59 & 411603.7 & 27143 & 31287.2 & 198.7 & $1.54 \mathrm{e}+38$ & & & r2-26 \\
\hline 35 & 67.8 & 004248.53 & 411521.2 & 12231 & 14353.6 & 133.8 & $6.74 e+37$ & & & $\mathrm{r} 1-1$ \\
\hline 36 & 72.2 & 004250.62 & 411557.1 & 35 & 23.7 & 8.5 & $1.15 \mathrm{e}+35$ & SNR & B90 101 & r2-56 \\
\hline 37 & 73.4 & 004239.65 & 411700.7 & 35 & 33.5 & 8.4 & $1.71 \mathrm{e}+35$ & & & K \\
\hline 38 & 73.6 & 004245.22 & 411722.3 & 675 & 783.9 & 32.2 & $3.63 e+36$ & $\mathrm{t}$ & & r2-16 \\
\hline 39 & 75.5 & 004250.82 & 411551.6 & 25 & 22.1 & 7.4 & $1.08 \mathrm{e}+35$ & RNova & B68 27 & X \\
\hline 40 & 75.7 & 004238.80 & 411526.2 & 35 & 29.8 & 8.5 & $1.50 \mathrm{e}+35$ & & & r2-54 \\
\hline 41 & 76.0 & 004242.73 & 411455.5 & 46 & 47.2 & 9.4 & $2.55 e+35$ & $\mathrm{t}$ & & r2-20 \\
\hline 42 & 81.9 & 004246.14 & 411728.6 & 21 & 20.7 & 6.8 & $9.45 e+34$ & & & X \\
\hline 43 & 86.8 & 004242.34 & 411445.5 & 1868 & 2198.5 & 53.0 & $1.18 \mathrm{e}+37$ & & & r2-21 \\
\hline 44 & 89.2 & 004246.09 & 411736.3 & 67 & 74.8 & 11.1 & $3.41 e+35$ & GCC $t$ & Bol BH16 & r2-15 \\
\hline 45 & 90.5 & 004244.91 & 411739.7 & 771 & 901.5 & 34.4 & $4.13 e+36$ & & & r2-18 \\
\hline 46 & 97.2 & 004252.53 & 411540.0 & 2740 & 3237.8 & 63.9 & $1.51 \mathrm{e}+37$ & & & r2-12 \\
\hline 47 & 99.7 & 004252.44 & 411648.7 & 140 & 158.6 & 15.4 & $7.52 \mathrm{e}+35$ & $\mathrm{t}$ & & X \\
\hline 48 & 107.5 & 004249.15 & $41 \quad 1742.0$ & 90 & 103.4 & 12.6 & $4.75 e+35$ & & & r2-41 \\
\hline 49 & 113.5 & 004244.63 & 411802.8 & 21 & 19.9 & 6.8 & $9.00 e+34$ & & & X \\
\hline 50 & 114.3 & 004239.54 & 411428.5 & 1169 & 1370.6 & 42.1 & $6.79 e+36$ & & & r2-25 \\
\hline 51 & 116.9 & 004234.78 & 411523.3 & 149 & 167.8 & 15.9 & $8.27 e+35$ & $\mathrm{t}$ & & r2-28 \\
\hline 52 & 117.8 & 004233.90 & 411619.8 & 1217 & 1426.2 & 42.9 & $7.25 e+36$ & & & r2-30 \\
\hline 53 & 119.1 & 004239.27 & 411424.7 & 48 & 40.9 & 9.7 & $2.50 \mathrm{e}+35$ & & & r2-62 \\
\hline 54 & 120.0 & 004254.94 & 411603.2 & 11609 & 13667.2 & 130.1 & $6.21 e+37$ & & & r2-11 \\
\hline 55 & 122.6 & 004245.10 & 411407.1 & 618 & 720.8 & 31.0 & $3.48 \mathrm{e}+36$ & & & r2-17 \\
\hline 56 & 124.3 & 004252.30 & 411735.0 & 122 & 137.6 & 14.5 & $6.37 e+35$ & & & r2-50 \\
\hline 57 & 126.2 & 004242.63 & 411404.6 & 24 & 20.3 & 7.3 & $1.04 \mathrm{e}+35$ & & & $\mathrm{X}$ \\
\hline 58 & 130.6 & 004236.05 & 411741.0 & 15 & 13.0 & 6.0 & $6.04 e+34$ & GCC & Bol B261 & $X$ \\
\hline 59 & 134.3 & 004233.41 & 411703.5 & 196 & 225.7 & 18.0 & $1.11 \mathrm{e}+36$ & $\mathrm{t}$ & & r2-70 \\
\hline 60 & 134.9 & 004232.53 & 411545.7 & 93 & 104.1 & 12.8 & $4.91 e+35$ & & & $\mathrm{r} 2-55$ \\
\hline 61 & 138.2 & 004249.24 & 411816.0 & 1627 & 1915.2 & 49.5 & $8.74 \mathrm{e}+36$ & & & r2-14 \\
\hline 62 & 140.6 & 004250.25 & $\begin{array}{llll}41 & 18 & 13.1\end{array}$ & 24 & 23.5 & 7.2 & $1.10 \mathrm{e}+35$ & & & r2-40 \\
\hline 63 & 140.6 & 004240.56 & 411355.3 & 21 & 15.6 & 6.9 & $7.59 e+34$ & & & r2-23 \\
\hline 64 & 148.9 & 004231.14 & 411621.7 & 7403 & 8689.1 & 104.2 & $5.21 e+37$ & & & r2-34 \\
\hline
\end{tabular}


Table 2. continued.

\begin{tabular}{|c|c|c|c|c|c|c|c|c|c|c|}
\hline $\begin{array}{l}\text { Number } \\
\text { (1) }\end{array}$ & $\begin{array}{l}\text { distance } \\
\text { (2) }\end{array}$ & $\begin{array}{l}\text { RA } \\
(3)\end{array}$ & $\begin{array}{l}\text { Dec } \\
\text { (4) }\end{array}$ & $\begin{array}{l}\text { cts } \\
\text { (5) }\end{array}$ & $\begin{array}{r}\text { corrected cts } \\
(6)\end{array}$ & $\begin{array}{r}\text { error } \\
(7)\end{array}$ & $\begin{array}{l}\text { luminosity } \\
\text { (8) }\end{array}$ & $\begin{array}{l}\text { type } \\
\text { (9) }\end{array}$ & $\begin{array}{l}\text { id } \\
\text { (10) }\end{array}$ & $\begin{array}{c}\text { name } \\
\text { (11) }\end{array}$ \\
\hline 65 & 161.5 & 004258.12 & 411652.5 & 21 & 18.6 & 6.9 & $8.64 \mathrm{e}+34$ & GCC & Bol AU010 & $\mathrm{X}$ \\
\hline 66 & 162.5 & 004240.22 & 411845.2 & 1090 & 1278.4 & 40.8 & $6.15 \mathrm{e}+36$ & & & r2-24 \\
\hline 67 & 163.0 & 004258.32 & 411529.2 & 1537 & 1807.0 & 48.2 & $8.39 e+36$ & & & r2-7 \\
\hline 68 & 163.9 & 004234.45 & 411809.8 & 94 & 105.3 & 12.9 & $5.01 \mathrm{e}+35$ & $\mathrm{t}$ & & r2-29 \\
\hline 69 & 163.9 & 004236.61 & 411350.3 & 130 & 144.9 & 14.9 & $7.06 \mathrm{e}+35$ & & & r2-42 \\
\hline 70 & 164.1 & 004230.28 & 411653.2 & 71 & 75.4 & 11.4 & $4.64 \mathrm{e}+35$ & & & r2-44 \\
\hline 71 & 167.1 & 004240.67 & 411327.4 & 1530 & 1803.7 & 48.1 & $8.75 e+36$ & & & r2-22 \\
\hline 72 & 170.2 & 004243.31 & 411319.6 & 844 & 991.6 & 36.0 & $5.02 \mathrm{e}+36$ & $\mathrm{t}$ & & r2-19 \\
\hline 73 & 172.4 & 004252.53 & 411834.8 & 59 & 63.8 & 10.5 & $3.06 \mathrm{e}+35$ & & & r2-49 \\
\hline 74 & 175.5 & 004259.87 & 411605.8 & 6988 & 8269.1 & 101.4 & $3.76 e+37$ & GC & Bol B144 & r2-5 \\
\hline 75 & 177.1 & 004250.72 & 411327.8 & 20 & 18.4 & 6.7 & $9.03 e+34$ & & & $\mathrm{X}$ \\
\hline 76 & 185.8 & 004242.24 & 411913.8 & 37 & 38.7 & 8.6 & $1.83 e+35$ & RAD & B90 86 & r2-53 \\
\hline 77 & 186.3 & 004252.64 & 411328.5 & 60 & 61.4 & 10.6 & $2.98 \mathrm{e}+35$ & & & r2-38 \\
\hline 78 & 189.2 & 004252.53 & 411854.4 & 13139 & 15495.8 & 138.6 & $7.47 e+37$ & & & r2-13 \\
\hline 79 & 191.2 & 004255.19 & 411836.1 & 635 & 744.8 & 31.5 & $3.58 \mathrm{e}+36$ & & & r2-10 \\
\hline 80 & 192.4 & 004254.36 & 411333.9 & 30 & 28.1 & 7.9 & $1.36 \mathrm{e}+35$ & & & X \\
\hline 81 & 193.6 & 004255.62 & 411835.1 & 628 & 735.7 & 31.3 & $3.54 \mathrm{e}+36$ & FGS & Bol B138 & r2-9 \\
\hline 82 & 195.7 & 004229.84 & 411757.5 & 19 & 16.7 & 6.6 & $1.01 \mathrm{e}+35$ & GC & Bol B103 & X \\
\hline 83 & 204.1 & 004251.66 & 411302.9 & 260 & 297.7 & 20.6 & $1.51 \mathrm{e}+36$ & & & r2-39 \\
\hline 84 & 204.5 & 004227.49 & 411453.0 & 76 & 81.3 & 11.7 & $4.54 \mathrm{e}+35$ & $\mathrm{t}$ & & X \\
\hline 85 & 210.0 & 004256.93 & 411843.9 & 494 & 579.8 & 27.9 & $2.73 e+36$ & $\mathrm{t}$ & & r2-8 \\
\hline 86 & 211.4 & 004301.78 & 411726.4 & 44 & 46.1 & 9.3 & $2.39 \mathrm{e}+35$ & & & r2-46 \\
\hline 87 & 211.5 & 004242.82 & 411940.3 & 21 & 19.7 & 6.9 & $9.46 \mathrm{e}+34$ & & & X \\
\hline 88 & 215.2 & 004302.94 & 411522.6 & 4541 & 5335.8 & 82.2 & $2.52 \mathrm{e}+37$ & GC & Bol B146 & r2-4 \\
\hline 89 & 216.1 & 004226.57 & 411731.5 & 6 & 1.1 & 4.4 & $5.89 e+33$ & & & X \\
\hline 90 & 217.3 & 004303.22 & 411527.8 & 6398 & 7511.3 & 97.2 & $3.57 e+37$ & $\mathrm{t}$ & & r2-3 \\
\hline 91 & 217.5 & 004258.09 & 411337.3 & 39 & 36.6 & 8.9 & $1.72 \mathrm{e}+35$ & & & X \\
\hline 92 & 221.0 & 004232.75 & 411310.9 & 1005 & 1131.2 & 39.3 & $6.11 \mathrm{e}+36$ & & & r2-31 \\
\hline 93 & 222.8 & 004232.09 & 411314.4 & 5162 & 6067.4 & 87.3 & $3.18 \mathrm{e}+37$ & & & r2-32 \\
\hline 94 & 222.8 & 004249.03 & 411945.8 & 31 & 28.6 & 8.0 & $1.41 \mathrm{e}+35$ & RAD & SIM WSTB 37W138 & r2-66 \\
\hline 95 & 224.9 & 004304.25 & 411601.3 & 969 & 1139.6 & 38.6 & $5.62 \mathrm{e}+36$ & GCC & Fan 42 & r2-1 \\
\hline 96 & 229.2 & 004224.19 & 411536.9 & 35 & 26.1 & 8.5 & $1.50 \mathrm{e}+35$ & & & r2-52 \\
\hline 97 & 229.6 & 004232.09 & 411913.1 & 37 & 35.0 & 8.7 & $1.70 \mathrm{e}+35$ & & & X \\
\hline 98 & 230.3 & 004258.10 & 411319.6 & 34 & 31.8 & 8.4 & $1.50 \mathrm{e}+35$ & & & $X$ \\
\hline 99 & 232.6 & 004301.71 & 411814.5 & 31 & 26.6 & 8.0 & $1.33 \mathrm{e}+35$ & & & $\mathrm{r} 2-47$ \\
\hline 100 & 234.4 & 004301.12 & 411351.6 & 242 & 270.6 & 19.9 & $1.27 \mathrm{e}+36$ & & & r2-37 \\
\hline 101 & 235.1 & 004230.96 & 411910.1 & 104 & 117.9 & 13.5 & $6.95 \mathrm{e}+35$ & & & r2-43 \\
\hline 102 & 240.7 & 004224.24 & 411731.5 & 44 & 38.9 & 9.3 & $2.19 \mathrm{e}+35$ & & & r2-57 \\
\hline 103 & 243.2 & 004222.96 & 411535.3 & 13877 & 16254.1 & 142.4 & $9.59 e+37$ & & & r3-39 \\
\hline 104 & 244.6 & 004301.72 & 411835.5 & 28 & 24.1 & 7.7 & $1.24 \mathrm{e}+35$ & & & X \\
\hline 105 & 246.8 & 004305.68 & 411702.7 & 18818 & 21892.3 & 166.0 & $1.05 \mathrm{e}+38$ & $\mathrm{t}$ & & r2-67 \\
\hline 106 & 248.9 & 004303.87 & 411804.9 & 4658 & 5511.9 & 83.1 & $2.81 \mathrm{e}+37$ & $\mathrm{GC}$ & Bol B148 & r2-2 \\
\hline 107 & 251.0 & 004244.40 & 411158.4 & 488 & 571.2 & 27.7 & $4.36 \mathrm{e}+36$ & & & r3-30 \\
\hline 108 & 253.7 & 004247.24 & 411157.9 & 159 & 181.2 & 16.4 & $1.34 \mathrm{e}+36$ & & & r3-27 \\
\hline 109 & 254.3 & 004224.16 & 411415.3 & 22 & 19.9 & 7.0 & $1.21 \mathrm{e}+35$ & & & X \\
\hline 110 & 255.9 & 004231.26 & 411938.9 & 2884 & 3395.5 & 65.6 & $2.01 \mathrm{e}+37$ & GC & Bol B107 & r2-33 \\
\hline 111 & 256.9 & 004259.66 & 411919.3 & 6646 & 7851.7 & 98.9 & $3.94 \mathrm{e}+37$ & GC & Bol B143 & r2-6 \\
\hline 112 & 257.4 & 004221.49 & 411601.3 & 4270 & 5026.6 & 79.5 & $3.20 \mathrm{e}+37$ & & & r3-42 \\
\hline 113 & 257.6 & 004235.22 & 412005.7 & 1496 & 1753.5 & 47.6 & $9.10 \mathrm{e}+36$ & & & $\mathrm{r} 2-27$ \\
\hline 114 & 259.1 & 004302.92 & 411841.5 & 22 & 14.3 & 7.1 & $7.30 \mathrm{e}+34$ & & & X \\
\hline 115 & 260.3 & 004221.29 & 411548.8 & 21 & 15.5 & 6.9 & $1.09 \mathrm{e}+35$ & RAD & B90 65 & $\mathrm{X}$ \\
\hline 116 & 262.3 & 004225.15 & 411340.6 & 614 & 712.8 & 31.0 & $4.44 \mathrm{e}+36$ & & & $\mathrm{r} 2-45$ \\
\hline 117 & 262.5 & 004221.80 & 411502.7 & 32 & 29.1 & 8.1 & $1.89 e+35$ & & & X \\
\hline 118 & 266.2 & 004256.04 & 411218.4 & 537 & 634.5 & 29.0 & $4.53 e+36$ & $\mathrm{t}$ & & r2-71 \\
\hline 119 & 267.5 & 004248.29 & 412033.1 & 24 & 22.6 & 7.2 & $1.11 \mathrm{e}+35$ & & & X \\
\hline 120 & 267.9 & 004223.16 & 411407.5 & 1223 & 1435.2 & 43.2 & $8.95 \mathrm{e}+36$ & & & r3-38 \\
\hline 121 & 269.0 & 004259.52 & 411242.3 & 173 & 191.7 & 17.1 & $9.62 \mathrm{e}+35$ & & & r2-48 \\
\hline 122 & 270.0 & 004254.79 & 412012.2 & 21 & 17.9 & 6.9 & $8.95 e+34$ & & & $\mathrm{X}$ \\
\hline 123 & 271.5 & 004244.85 & 411138.0 & 2955 & 3465.1 & 66.5 & $2.53 \mathrm{e}+37$ & & & r3-29 \\
\hline 124 & 271.9 & 004257.17 & 411959.5 & 24 & 19.7 & 7.3 & $9.79 e+34$ & & & X \\
\hline 125 & 277.1 & 004226.05 & 411915.0 & 1447 & 1697.3 & 46.9 & $9.40 \mathrm{e}+36$ & GC & Bol B096 & r2-36 \\
\hline 126 & 278.9 & 004221.57 & 411419.7 & 684 & 800.4 & 32.6 & $5.30 \mathrm{e}+36$ & & & r3-41 \\
\hline 127 & 279.8 & 004231.32 & 412007.9 & 111 & 121.3 & 13.9 & $7.27 \mathrm{e}+35$ & & & r2-51 \\
\hline 128 & 284.0 & 004307.12 & 411810.2 & 166 & 184.7 & 16.8 & $9.91 \mathrm{e}+35$ & Nova $\mathrm{t}$ & PIE RJC 99 Jul 98 & r3-115 \\
\hline 129 & 285.4 & 004220.85 & 411756.7 & 33 & 31.4 & 8.2 & $1.85 \mathrm{e}+35$ & & & $\mathrm{X}$ \\
\hline
\end{tabular}


Table 2. continued.

\begin{tabular}{|c|c|c|c|c|c|c|c|c|c|c|}
\hline $\begin{array}{l}\text { Number } \\
\text { (1) }\end{array}$ & $\begin{array}{l}\text { distance } \\
(2)\end{array}$ & $\begin{array}{l}\text { RA } \\
(3) \\
\end{array}$ & $\begin{array}{c}\text { Dec } \\
(4)\end{array}$ & $\begin{array}{l}\text { cts } \\
(5)\end{array}$ & $\begin{array}{r}\text { corrected cts } \\
\text { (6) }\end{array}$ & $\begin{array}{r}\text { error } \\
(7) \\
\end{array}$ & $\begin{array}{c}\text { luminosity } \\
(8)\end{array}$ & $\begin{array}{l}\text { type } \\
(9)\end{array}$ & $\begin{array}{l}\text { id } \\
\text { (10) }\end{array}$ & $\begin{array}{c}\text { name } \\
(11)\end{array}$ \\
\hline 130 & 287.6 & 004221.09 & 411808.6 & 81 & 87.7 & 12.1 & $5.13 e+35$ & $\mathrm{t}$ & & r3-43 \\
\hline 131 & 289.5 & 004228.30 & 411223.1 & 6286 & 7379.4 & 96.2 & $5.72 \mathrm{e}+37$ & & & r2-35 \\
\hline 132 & 291.5 & 004222.44 & 411334.1 & 2253 & 2642.2 & 58.2 & $1.70 \mathrm{e}+37$ & & & r3-40 \\
\hline 133 & 294.7 & 004233.82 & 412039.3 & 27 & 25.3 & 7.6 & $1.33 e+35$ & & & $\mathrm{X}$ \\
\hline 134 & 297.5 & 004258.61 & 411159.5 & 28 & 26.5 & 7.7 & $1.72 \mathrm{e}+35$ & & & r2-59 \\
\hline 135 & 297.6 & 004241.65 & 412105.5 & 450 & 522.2 & 26.7 & $3.64 \mathrm{e}+36$ & & & r3-31 \\
\hline 136 & 298.2 & 004247.83 & 411113.9 & 168 & 189.4 & 16.8 & $1.50 \mathrm{e}+36$ & GCC $\mathrm{t}$ & Bol B128 & X \\
\hline 137 & 300.6 & 004259.02 & 411158.8 & 33 & 32.8 & 8.2 & $2.14 \mathrm{e}+35$ & & & r2-58 \\
\hline 138 & 301.6 & 004220.85 & 411344.5 & 27 & 23.2 & 7.6 & $1.62 \mathrm{e}+35$ & & & X \\
\hline 139 & 306.8 & 004310.62 & 411451.4 & 14308 & 16890.8 & 144.8 & $8.99 \mathrm{e}+37$ & $\mathrm{GC}$ & Bol B153 & r3-15 \\
\hline 140 & 307.1 & 004250.02 & 411109.1 & 93 & 98.7 & 12.9 & $7.51 \mathrm{e}+35$ & & & r3-24 \\
\hline 141 & 308.9 & 004241.12 & 411102.6 & 30 & 25.9 & 8.0 & $2.07 \mathrm{e}+35$ & & & r3-32 \\
\hline 142 & 311.2 & 004246.94 & $\begin{array}{lll}41 & 21 & 19.2\end{array}$ & 214 & 243.8 & 18.8 & $1.71 \mathrm{e}+36$ & & & r3-28 \\
\hline 143 & 311.9 & 004218.43 & 411759.6 & 8 & 0.8 & 4.9 & $5.37 \mathrm{e}+33$ & & & X \\
\hline 144 & 312.2 & 004306.80 & 411911.6 & 89 & 83.8 & 12.7 & $3.98 \mathrm{e}+35$ & EXT & Source 231 & r3-67 \\
\hline 145 & 312.9 & 004216.55 & 411610.7 & 30 & 27.5 & 7.9 & $1.90 \mathrm{e}+35$ & & & r3-75 \\
\hline 146 & 313.5 & 004217.04 & 411508.2 & 261 & 299.0 & 20.6 & $2.19 \mathrm{e}+36$ & $\mathrm{t}$ & & r3-46 \\
\hline 147 & 314.6 & 004306.75 & 411916.6 & 100 & 97.3 & 13.3 & $4.73 e+35$ & EXT & Source 160 & r3-67 \\
\hline 148 & 316.2 & 004218.65 & 411401.9 & 5388 & 6324.4 & 89.2 & $4.69 e+37$ & $\mathrm{GC}$ & Bol B086 & r3-44 \\
\hline 149 & 318.6 & 004216.09 & 411553.3 & 37 & 36.6 & 8.6 & $2.80 \mathrm{e}+35$ & & & r3-76 \\
\hline 150 & 318.9 & 004247.88 & 411053.1 & 54 & 56.4 & 10.1 & $4.71 e+35$ & & & r3-26 \\
\hline 151 & 320.1 & 004302.44 & 411203.1 & 54 & 55.6 & 10.1 & $4.05 e+35$ & & & r3-68 \\
\hline 152 & 321.4 & 004220.48 & 411313.2 & 34 & 27.6 & 8.4 & $2.03 e+35$ & & & r3-89 \\
\hline 153 & 327.9 & 004311.37 & 411809.9 & 317 & 360.6 & 22.7 & $1.93 e+36$ & & & r3-14 \\
\hline 154 & 330.4 & 004215.69 & 411721.0 & 2105 & 2476.8 & 56.2 & $1.69 \mathrm{e}+37$ & & & r3-47 \\
\hline 155 & 335.1 & 004309.85 & 411900.9 & 1328 & 1559.0 & 45.0 & $8.29 \mathrm{e}+36$ & $\mathrm{GCC} \mathrm{t}$ & Fan 44 & r3-16 \\
\hline 156 & 335.6 & 004227.71 & 412048.1 & 68 & 68.7 & 11.2 & $4.54 \mathrm{e}+35$ & & & r3-37 \\
\hline 157 & 338.5 & 004240.68 & 411033.4 & 154 & 167.0 & 16.2 & $1.35 \mathrm{e}+36$ & GC & Bol B123 & r3-34 \\
\hline 158 & 339.3 & $0043 \quad 13.88$ & $41 \quad 1712.2$ & 70 & 64.0 & 11.4 & $3.59 \mathrm{e}+35$ & & & r3-12 \\
\hline 159 & 340.2 & 004308.64 & 411248.4 & 1109 & 1292.5 & 41.2 & $6.86 e+36$ & & & r3-17 \\
\hline 160 & 341.1 & 004257.91 & 411104.8 & 4128 & 4861.9 & 78.4 & $3.65 e+37$ & & & r3-22 \\
\hline 161 & 341.5 & 004314.38 & 411650.2 & 119 & 121.6 & 14.5 & $6.61 \mathrm{e}+35$ & & & r3-11 \\
\hline 162 & 343.1 & 004250.76 & 411034.1 & 48 & 46.0 & 9.7 & $3.58 \mathrm{e}+35$ & GCC & Bol BH18 & r3-71 \\
\hline 163 & 344.5 & 004303.02 & 412042.0 & 302 & 344.7 & 22.1 & $1.96 \mathrm{e}+36$ & $\mathrm{PN}$ & CIA 165 & r3-21 \\
\hline 164 & 344.7 & 004252.26 & 412142.3 & 27 & 23.7 & 7.6 & $1.75 \mathrm{e}+35$ & & & X \\
\hline 165 & 346.2 & 004215.24 & 411801.3 & 190 & 210.4 & 17.8 & $1.48 \mathrm{e}+36$ & & & r3-49 \\
\hline 166 & 348.8 & 004313.23 & 411813.5 & 418 & 473.5 & 25.8 & $2.67 e+36$ & RNova & CFN 26 & r3-13 \\
\hline 167 & 350.4 & 004216.98 & 411856.4 & 54 & 51.6 & 10.2 & $3.24 \mathrm{e}+35$ & & & r3-91 \\
\hline 168 & 350.6 & 004233.46 & 412138.0 & 49 & 45.2 & 9.8 & $3.83 e+35$ & FGS & Bol B113 & X \\
\hline 169 & 352.7 & 004213.07 & 411627.9 & 79 & 80.3 & 12.0 & $5.72 \mathrm{e}+35$ & & & r3-53 \\
\hline 170 & 358.9 & 004234.17 & 412149.7 & 267 & 302.6 & 20.9 & $2.38 \mathrm{e}+36$ & & & r3-35 \\
\hline 171 & 361.8 & 004316.35 & 411630.5 & 30 & 20.4 & 8.0 & $1.39 \mathrm{e}+35$ & & & X \\
\hline 172 & 362.0 & 004307.50 & 412019.9 & 550 & 632.5 & 29.4 & $3.51 \mathrm{e}+36$ & GCC & Bol B150 & r3-18 \\
\hline 173 & 366.3 & 004240.73 & 411005.3 & 33 & 25.8 & 8.3 & $2.24 \mathrm{e}+35$ & & & X \\
\hline 174 & 366.5 & 004211.99 & 411648.7 & 402 & 458.1 & 25.3 & $3.31 \mathrm{e}+36$ & & & r3-55 \\
\hline 175 & 368.7 & 004240.92 & 412216.1 & 52 & 48.9 & 10.0 & $3.51 \mathrm{e}+35$ & & & r3-33 \\
\hline 176 & 369.4 & 004219.01 & 412004.3 & 63 & 58.3 & 10.9 & $3.53 e+35$ & & & r3-90 \\
\hline 177 & 369.5 & 004218.37 & 411223.8 & 3119 & 3651.3 & 68.2 & $3.50 \mathrm{e}+37$ & & & r3-45 \\
\hline 178 & 372.3 & 004301.70 & 411052.9 & 48 & 43.8 & 9.7 & $3.55 e+35$ & & & r3-96 \\
\hline 179 & 378.3 & 004212.18 & 411758.7 & 1554 & 1800.7 & 48.5 & $1.20 \mathrm{e}+37$ & GCC & Bol B078 & r3-54 \\
\hline 180 & 378.6 & 004303.31 & 412121.7 & 1486 & 1745.3 & 47.5 & $1.34 \mathrm{e}+37$ & FGS & Bol B147 & r3-19 \\
\hline 181 & 380.7 & 004213.16 & 411836.5 & 6027 & 7031.2 & 94.3 & $4.91 \mathrm{e}+37$ & FGS & Cra 13 & r3-52 \\
\hline 182 & 383.1 & 004315.06 & 411326.6 & 44 & 31.8 & 9.4 & $1.82 \mathrm{e}+35$ & & & X \\
\hline 183 & 385.5 & 004314.18 & 411302.0 & 51 & 35.7 & 10.0 & $2.04 \mathrm{e}+35$ & & & $X$ \\
\hline 184 & 388.1 & 004210.29 & 411509.9 & 555 & 640.8 & 29.5 & $4.99 e+36$ & & & r3-58 \\
\hline 185 & 389.2 & $0043 \quad 16.10$ & 411841.3 & 411 & 469.3 & 25.6 & $3.35 \mathrm{e}+36$ & & & r3-9 \\
\hline 186 & 390.0 & 004249.36 & 412235.2 & 45 & 38.4 & 9.4 & $2.99 e+35$ & & & $\mathrm{X}$ \\
\hline 187 & 392.9 & 004215.14 & 411234.6 & 2746 & 3194.3 & 64.2 & $2.67 e+37$ & & & r3-50 \\
\hline 188 & 393.2 & 004225.35 & $41 \quad 1039.4$ & 33 & 16.3 & 8.4 & $1.59 \mathrm{e}+35$ & & & $X$ \\
\hline 189 & 396.2 & 004314.60 & 411930.5 & 61 & 49.2 & 10.8 & $2.82 \mathrm{e}+35$ & & & $\mathrm{X}$ \\
\hline 190 & 403.9 & 004209.51 & 411745.6 & 1077 & 1244.1 & 40.6 & $8.92 \mathrm{e}+36$ & GCC & Mita 140 & r3-59 \\
\hline 191 & 404.3 & 004254.26 & 410941.0 & 29 & 22.5 & 7.9 & $1.91 \mathrm{e}+35$ & & & X \\
\hline 192 & 411.3 & 004228.22 & 411000.4 & 1989 & 2316.9 & 54.8 & $2.45 \mathrm{e}+37$ & & & r3-36 \\
\hline 193 & 411.5 & 004212.69 & 411244.1 & 62 & 51.9 & 10.9 & $4.14 e+35$ & & & r3-92 \\
\hline
\end{tabular}


Table 2. continued.

\begin{tabular}{|c|c|c|c|c|c|c|c|c|c|c|}
\hline $\begin{array}{c}\text { Number } \\
\text { (1) }\end{array}$ & $\begin{array}{l}\text { distance } \\
\text { (2) }\end{array}$ & $\begin{array}{l}\text { RA } \\
\text { (3) }\end{array}$ & $\begin{array}{l}\text { Dec } \\
\text { (4) }\end{array}$ & $\begin{array}{l}\text { cts } \\
\text { (5) }\end{array}$ & $\begin{array}{r}\text { corrected cts } \\
\text { (6) }\end{array}$ & $\begin{array}{r}\text { error } \\
(7)\end{array}$ & $\begin{array}{c}\text { luminosity } \\
(8)\end{array}$ & $\begin{array}{l}\text { type } \\
\text { (9) }\end{array}$ & $\begin{array}{l}\text { id } \\
\text { (10) }\end{array}$ & $\begin{array}{c}\text { name } \\
(11)\end{array}$ \\
\hline 194 & 411.8 & 004312.35 & 412033.5 & 51 & 31.7 & 10.1 & $1.85 \mathrm{e}+35$ & & & $X$ \\
\hline 195 & 412.7 & 004303.13 & 411015.6 & 561 & 643.6 & 29.7 & $5.25 \mathrm{e}+36$ & GCC & Fan 37 & r3-20 \\
\hline 196 & 417.2 & 004215.51 & 412031.5 & 570 & 655.6 & 29.9 & $4.67 e+36$ & GCC & Fan 16 & r3-48 \\
\hline 197 & 420.2 & 004321.57 & 411557.4 & 52 & 37.0 & 10.1 & $2.37 \mathrm{e}+35$ & & & r3-104 \\
\hline 198 & 425.4 & 004207.10 & 411720.1 & 140 & 145.1 & 15.5 & $1.10 \mathrm{e}+36$ & & & r3-79 \\
\hline 199 & 426.1 & 004211.00 & 411248.3 & 270 & 296.3 & 21.0 & $2.34 \mathrm{e}+36$ & & & r3-57 \\
\hline 200 & 426.6 & 004321.08 & 411750.5 & 724 & 826.5 & 33.6 & $5.02 \mathrm{e}+36$ & & & r3-7 \\
\hline 201 & 430.6 & 004207.77 & $\begin{array}{llll}41 & 18 & 14.9\end{array}$ & 4275 & 4995.6 & 79.7 & $3.72 \mathrm{e}+37$ & & & r3-61 \\
\hline 202 & 432.2 & 004317.06 & 411225.1 & 28 & 19.9 & 7.8 & $1.75 \mathrm{e}+35$ & & & X \\
\hline 203 & 438.5 & 004232.04 & 412305.5 & 68 & 66.5 & 11.3 & $5.82 \mathrm{e}+35$ & & & r3-86 \\
\hline 204 & 441.7 & 004219.73 & 412153.5 & 93 & 90.4 & 13.0 & $8.51 \mathrm{e}+35$ & GCC & Mita 166 & r3-74 \\
\hline 205 & 443.4 & 004320.92 & 411851.7 & 97 & 87.1 & 13.2 & $5.20 \mathrm{e}+35$ & & & r3-66 \\
\hline 206 & 448.6 & 004259.00 & 410912.6 & 43 & 32.3 & 9.3 & $2.76 e+35$ & & & X \\
\hline 207 & 448.9 & 004319.98 & 411250.2 & 50 & 32.3 & 10. & $2.18 \mathrm{e}+35$ & & & $X$ \\
\hline 208 & 451.6 & 004315.42 & 411125.3 & 167 & 182.8 & 16.8 & $1.57 \mathrm{e}+36$ & $\mathrm{GC}$ & Bol B161 & r3-10 \\
\hline 209 & 453.6 & 004208.21 & 411249.6 & 90 & 82.6 & 12.8 & $6.90 \mathrm{e}+35$ & & & r3-93 \\
\hline 210 & 453.8 & 004215.06 & 412121.4 & 55 & 46.2 & 10.3 & $5.13 e+35$ & GCC & Fan 15 & r3-51 \\
\hline 211 & 454.4 & 004204.14 & 411532.6 & 164 & 174.2 & 16.7 & $1.42 \mathrm{e}+36$ & & & r3-62 \\
\hline 212 & 457.2 & 004233.90 & 412331.3 & 160 & 168.9 & 16.5 & $1.34 \mathrm{e}+36$ & $\mathrm{t}$ & & X \\
\hline 213 & 458.9 & 004209.62 & 412009.8 & 76 & 68.7 & 11.9 & $5.43 e+35$ & RAD & B90 34 & r3-102 \\
\hline 214 & 461.6 & 004318.89 & 412017.0 & 170 & 169.1 & 17.0 & $1.07 e+36$ & & & r3-8 \\
\hline 215 & 463.3 & 004324.82 & 411727.3 & 308 & 335.5 & 22.4 & $2.11 \mathrm{e}+36$ & & & r3-6 \\
\hline 216 & 463.5 & 004205.72 & $\begin{array}{lll}41 & 13 & 29.9\end{array}$ & 64 & 45.6 & 11.1 & $3.86 e+35$ & $\mathrm{t}$ & & r3-125 \\
\hline 217 & 464.3 & 004319.54 & 412010.1 & 94 & 80.2 & 13.1 & $4.93 e+35$ & & & $X$ \\
\hline 218 & 468.0 & 004306.65 & 412243.9 & 87 & 87.3 & 12.6 & $7.83 e+35$ & EmO & MLA 686 & r3-83 \\
\hline 219 & 468.4 & 004318.39 & 411141.8 & 38 & 29.3 & 8.8 & $2.69 e+35$ & & & X \\
\hline 220 & 469.6 & 004322.34 & 411258.3 & 61 & 42.5 & 10.8 & $2.76 e+35$ & & & r3-82 \\
\hline 221 & 479.9 & 004300.24 & 410844.4 & 48 & 41.6 & 9.7 & $4.33 e+35$ & & & $\mathrm{X}$ \\
\hline 222 & 480.5 & 004317.95 & 411114.7 & 42 & 33.0 & 9.2 & $3.33 e+35$ & & & $X$ \\
\hline 223 & 480.7 & 004248.95 & 412407.3 & 109 & 106.5 & 13.9 & $6.95 e+35$ & & & r3-84 \\
\hline 224 & 482.1 & 004324.13 & 411314.3 & 79 & 60.5 & 12.1 & $4.02 \mathrm{e}+35$ & & & r3-65 \\
\hline 225 & 482.6 & 004216.13 & 412212.9 & 41 & 28.6 & 9.1 & $3.88 \mathrm{e}+35$ & & & X \\
\hline 226 & 484.8 & 004209.10 & 412048.0 & 698 & 799.3 & 33.0 & $7.81 \mathrm{e}+36$ & & & r3-60 \\
\hline 227 & 487.2 & 004211.78 & 411049.0 & 386 & 430.9 & 24.9 & $6.10 \mathrm{e}+36$ & & & r3-56 \\
\hline 228 & 493.0 & 004318.73 & 412113.8 & 48 & 41.3 & 9.7 & $4.07 e+35$ & & & X \\
\hline 229 & 504.0 & 004245.80 & 412433.1 & 96 & 91.9 & 13.1 & $6.76 e+35$ & & & r3-72 \\
\hline 230 & 507.5 & 004326.34 & 411911.6 & 151 & 152.5 & 16.1 & $1.25 \mathrm{e}+36$ & RAD & GLG 005 & r3-64 \\
\hline 231 & 511.2 & 004327.92 & 411829.9 & 583 & 654.2 & 30.3 & $5.39 \mathrm{e}+36$ & SNR & B90 142 & r3-63 \\
\hline 232 & 513.6 & 004205.90 & 411133.7 & 85 & 75.6 & 12.5 & $1.10 \mathrm{e}+36$ & & & r3-80 \\
\hline 233 & 521.7 & 004224.51 & 412401.1 & 58 & 43.6 & 10.6 & $4.12 \mathrm{e}+35$ & & & X \\
\hline 234 & 529.2 & 004310.00 & 412332.5 & 62 & 51.3 & 10.8 & $5.89 e+35$ & $\mathrm{PN} \mathrm{t}$ & CIA 350 & r3-127 \\
\hline 235 & 537.6 & 004207.58 & $\begin{array}{lll}41 & 10 & 27.1\end{array}$ & 104 & 93.0 & 13.7 & $1.45 \mathrm{e}+36$ & & & r3-94 \\
\hline 236 & 548.6 & 004240.98 & 410702.0 & 59 & 49.5 & 10.6 & $7.54 \mathrm{e}+35$ & & & r3-85 \\
\hline 237 & 554.4 & 004248.50 & 412521.8 & 4302 & 5020.8 & 80.1 & $3.91 \mathrm{e}+37$ & $\mathrm{t}$ & & r3-25 \\
\hline 238 & 565.0 & 004223.00 & 410738.2 & 135 & 122.1 & 15.4 & $2.06 \mathrm{e}+36$ & & & r3-73 \\
\hline 239 & 571.6 & 004333.83 & 411407.3 & 70 & 46.2 & 11.5 & $4.29 e+35$ & & & X \\
\hline 240 & 575.9 & 004249.41 & 410636.3 & 54 & 42.5 & 10.2 & $7.28 \mathrm{e}+35$ & & & r3-98 \\
\hline 241 & 585.2 & 004308.89 & 410734.2 & 38 & 34.9 & 8.8 & $8.72 \mathrm{e}+35$ & & & X \\
\hline 242 & 587.9 & 004334.32 & 411323.5 & 2185 & 2554.7 & 57.4 & $2.41 \mathrm{e}+37$ & & & r3-2 \\
\hline 243 & 589.9 & 004335.92 & 411432.8 & 446 & 431.6 & 27.0 & $2.54 \mathrm{e}+36$ & & & $X$ \\
\hline 244 & 590.7 & 004253.68 & 412550.6 & 313 & 322.0 & 22.6 & $3.66 \mathrm{e}+36$ & SNR & MG BA521 & r3-69 \\
\hline 245 & 600.3 & 004255.40 & 412556.5 & 1483 & 1696.3 & 47.6 & $1.84 \mathrm{e}+37$ & & & r3-23 \\
\hline 246 & 600.7 & 004151.65 & 411438.7 & 385 & 426.6 & 24.9 & $5.60 \mathrm{e}+36$ & & & r3-81 \\
\hline 247 & 603.5 & 004337.28 & 411443.4 & 5112 & 5992.7 & 87.0 & $5.65 e+37$ & GC & Bol B185 & r3-1 \\
\hline 248 & 617.2 & 004226.26 & 412552.2 & 581 & 660.6 & 30.2 & $9.26 \mathrm{e}+36$ & $\mathrm{EmO}$ & W2 & r3-87 \\
\hline 249 & 627.5 & 004150.35 & 411336.3 & 209 & 208.4 & 18.7 & $3.21 \mathrm{e}+36$ & & & r3-110 \\
\hline 250 & 627.5 & 004314.38 & 410721.6 & 1405 & 1650.1 & 46.2 & $6.12 e+37$ & $\mathrm{GC} \mathrm{t}$ & Bol B158 & r3-112 \\
\hline 251 & 630.4 & 004251.60 & 412634.5 & 95 & 100.0 & 13.0 & $1.47 \mathrm{e}+36$ & RAD & GLG 011 & r3-70 \\
\hline 252 & 634.1 & 004332.38 & 411041.0 & 1298 & 1503.3 & 44.5 & $3.12 \mathrm{e}+37$ & & & r3-3 \\
\hline
\end{tabular}


Table 2. continued.

\begin{tabular}{|c|c|c|c|c|c|c|c|c|c|c|}
\hline $\begin{array}{l}\text { Number } \\
\text { (1) }\end{array}$ & $\begin{array}{l}\text { distance } \\
\text { (2) }\end{array}$ & $\begin{array}{l}\text { RA } \\
\text { (3) }\end{array}$ & $\begin{array}{l}\text { Dec } \\
(4)\end{array}$ & $\begin{array}{l}\text { cts } \\
\text { (5) }\end{array}$ & $\begin{array}{r}\text { corrected cts } \\
(6)\end{array}$ & $\begin{array}{r}\text { error } \\
(7)\end{array}$ & $\begin{array}{c}\text { luminosity } \\
\text { (8) }\end{array}$ & $\begin{array}{l}\text { type } \\
\text { (9) }\end{array}$ & $\begin{array}{l}\text { id } \\
\text { (10) }\end{array}$ & $\begin{array}{l}\text { name } \\
(11)\end{array}$ \\
\hline 253 & 642.2 & 004314.59 & 412513.5 & 94 & 79.2 & 13.1 & $8.89 \mathrm{e}+35$ & $\mathrm{GC}$ & Bol B159 & r3-105 \\
\hline 254 & 645.6 & 004333.58 & 412139.1 & 70 & 73.3 & 11.4 & $1.38 \mathrm{e}+36$ & & & X \\
\hline 255 & 646.9 & 004237.97 & 410526.5 & 60 & 36.4 & 10.8 & $1.32 \mathrm{e}+36$ & & & r3-100 \\
\hline 256 & 660.4 & 004341.75 & 411400.9 & 79 & 50.7 & 12.2 & $5.81 \mathrm{e}+35$ & & & $X$ \\
\hline 257 & 669.7 & 004232.82 & 412706.5 & 26 & 18.0 & 7.5 & $3.02 \mathrm{e}+35$ & & & $X$ \\
\hline 258 & 676.4 & 004210.90 & 410647.8 & 151 & 103.2 & 16.4 & $1.95 \mathrm{e}+36$ & & & r3-78 \\
\hline 259 & 685.1 & 004220.57 & 412640.2 & 170 & 182.6 & 17.0 & $3.49 \mathrm{e}+36$ & $\mathrm{EmO}$ & W2 & K \\
\hline 260 & 691.1 & 004155.15 & 412302.8 & 87 & 57.2 & 12.8 & $2.40 \mathrm{e}+36$ & & & r3-108 \\
\hline 261 & 706.9 & 004343.94 & 411231.9 & 120 & 108.7 & 14.6 & $1.70 \mathrm{e}+36$ & RAD & B90 166 & X \\
\hline 262 & 715.3 & 004228.98 & 410435.4 & 785 & 807.6 & 35.1 & $2.45 e+37$ & & & r3-111 \\
\hline 263 & 718.2 & 004244.29 & 412807.6 & 84 & 71.5 & 12.4 & $1.17 \mathrm{e}+36$ & & & $\mathrm{X}$ \\
\hline
\end{tabular}

(1) - The sequence number; (2) - Distance to the centre in arcsec; (3),(4) - Right ascension and declination of source; (5) - Source counts; (6) - Source counts after background subtraction; (7) - Statistical error on source counts after background subtraction; (8) - X-ray luminosity, 0.5-8 keV, assuming $780 \mathrm{kpc}$ distance; (9) - Source Type: GC - confirmed globular cluster, GCC - globular cluster candidate, PN - planetary nebula, FGS - foreground star, Nova - nova, Rnova - Nova (random match), EmO - emission line object, RAD - radio source, SNR - supernova remnant, EXT - extended source, t - transient source; (10) - precise identification and reference: Bol - Galleti et al. (2004), Fan - Fan et al. (2005), Mita - Magnier (1993), MLA - Meyssonnier et al. (1993), W2 - Williams et al. (2004b), CIA - Ciardullo et al. (1989), CFN - Ciardullo et al. (1987), PIE - Pietsch et al. (2005a), SI - Shafter \& Irby (2001), B68 - Börngen (1968), SIM - Simbad, GLG - Gelfand et al. (2004), B90 Braun (1990), MG - Magnier et al. (1995), Cra - Crampton et al. (1984); (11) - Source name in Kong et al. (2002), Williams et al. (2004a) and Williams et al. (2006); Sources not included in these catalogues are marked with K if observed in Kaaret (2002), else with X, indicating that these are new sources. 